\title{
Separating Overlapped Fingerprints
}

\author{
Fanglin Chen, Jianjiang Feng, Member, IEEE, Anil K. Jain, Fellow, IEEE, Jie Zhou, Senior Member, IEEE, and \\ Jin Zhang
}

\begin{abstract}
Fingerprint images generally contain either a single fingerprint (e.g., rolled images) or a set of nonoverlapped fingerprints (e.g., slap fingerprints). However, there are situations where several fingerprints overlap on top of each other. Such situations are frequently encountered when latent (partial) fingerprints are lifted from crime scenes or residue fingerprints are left on fingerprint sensors. Overlapped fingerprints constitute a serious challenge to existing fingerprint recognition algorithms, since these algorithms are designed under the assumption that fingerprints have been properly segmented. In this paper, a novel algorithm is proposed to separate overlapped fingerprints into component or individual fingerprints. The basic idea is to first estimate the orientation field of the given image with overlapped fingerprints and then separate it into component orientation fields using a relaxation labeling technique. We also propose an algorithm to utilize fingerprint singularity information to further improve the separation performance. Experimental results indicate that the algorithm leads to good separation of overlapped fingerprints that leads to a significant improvement in the matching accuracy.
\end{abstract}

Index Terms-Fingerprint matching, fingerprint separation, latent fingerprints, orientation field, overlapped fingerprints, relaxation labeling, singularity.

\section{INTRODUCTION}

$\mathbf{F}$ INGERPRINT refers to the friction ridge skin on a human finger or its impression. A fingerprint is a type of oriented texture with locally smooth and intervening ridges and valleys. Fingerprints are claimed to be both unique and permanent, making it an ideal biometric trait for person identification [2]. In fact, fingerprint recognition has been used by law enforcement agencies all over the world to identify suspects and victims for more than a century.

Manuscript received September 23, 2010; revised December 19, 2010; accepted January 31, 2011. Date of publication February 14, 2011; date of current version May 18, 2011. This work was supported by the National $863 \mathrm{Hi}-\mathrm{Tech}$ Development Program of China under Grant 2008AA01Z123, and by the National Natural Science Foundation of China under Grant 60805017, Grant 61005023, Grant 61021063, and Grant 61020106004. The work of A. K. Jain was supported in part by WCU (World Class University) program funded by the Ministry of Education, Science and Technology through the National Research Foundation of Korea (R31-10008). An early version of this paper appeared in the Fourth IEEE International Conference on Biometrics: Theory, Applications and Systems (BTAS'10), September 2010. The associate editor coordinating the review of this manuscript and approving it for publication was Dr. Fabio Scotti.

F. Chen, J. Feng, J. Zhou, and J. Zhang are with the Department of Automation, Tsinghua University, Beijing 100084, China (e-mail: chenf106@mails.tsinghua.edu.cn; jfeng@tsinghua.edu.cn; jzhou@tsinghua.edu.cn; zhangjin02@mails.tsinghua.edu.cn).

A. K. Jain is with the Department of Computer Science and Engineering, Michigan State University, East Lansing, MI-48824 USA, and also with the Department of Brain and Cognitive Engineering, Korea University, Seoul 136713, Korea (e-mail: jain@cse.msu.edu).

Color versions of one or more of the figures in this paper are available online at http://ieeexplore.ieee.org.

Digital Object Identifier 10.1109/TIFS.2011.2114345

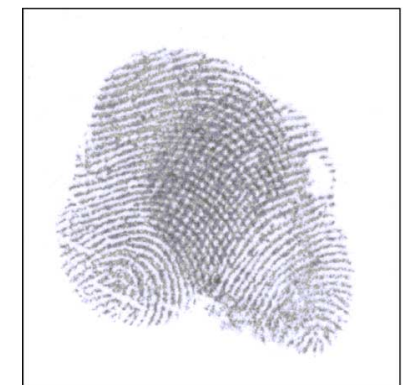

(a)

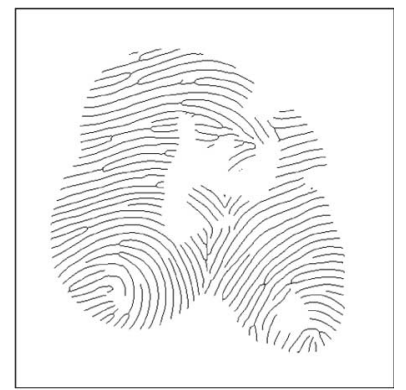

(b)
Fig. 1. (a) Overlapped fingerprint image and (b) its skeleton image extracted by a commercial fingerprint recognition algorithm.

Fingerprint matchers (manual or automatic) are mainly based on extracting and comparing characteristic points (minutiae) of ridges. As a result, reliable ridge extraction is very important for successful matching. Existing ridge extraction algorithms [3] work very well when ridge structures are well defined or the noise in fingerprint image is not significant. However, there exist many challenging situations, overlapped fingerprints being one of them [see Fig. 1(a)], where state-of-the-art matchers do not perform very well. Overlapped images are mainly encountered in latent fingerprints lifted from crime scenes [4]. When the same surface is touched by two fingers, the developed latent image may contain overlapped fingerprints. Overlapping may also occur in live-scan fingerprint images when the surface of fingerprint sensors contains the residue of fingerprints of previous users.

Fig. 1 shows an overlapped fingerprint image and its skeleton image extracted by a well-known commercial fingerprint recognition algorithm, VeriFinger 6.2 SDK [5]. As shown in Fig. 1(b), VeriFinger is unable to successfully segment the two component fingerprints and fails to extract ridges in the overlapped region. This example indicates that overlapped fingerprints constitute a serious challenge to the state-of-the-art fingerprint recognition algorithms. Manually marking features in overlapped fingerprints is also very difficult even for latent experts. Although a basic Fourier domain band-stop filtering technique can be used to remove overlapping repetitive lines in latent images [6], [7] separating overlapped fingerprints, which are not composed of straight lines, is much more complicated. Therefore, it is desirable to develop a technique that can automatically separate overlapped fingerprints into their individual fingerprints to improve the matching performance.

Forensic scientists have proposed a method to separate overlapped latent fingerprints using gold nanomaterial [8] during the latent development stage. While this technology is very interesting, it is not convenient and only works for a specific 
type of overlapped latent fingerprints, where component fingerprints are assumed to be covered with different lipids. A more universal and convenient solution is to develop an image processing algorithm to perform the separation task. Such an algorithm will not only benefit fingerprint recognition systems, but will also simplify manual feature marking as well. Fan et al. [9] proposed an algorithm to separate overlapped fingerprints based on image enhancement using a manually marked orientation field. However, it is very tedious and time-consuming for the user to manually mark the orientation field of each component fingerprint in the overlapped fingerprint image. Geng et al. [10] proposed to use morphological component analysis to separate overlapped fingerprints. However, experimental results showed that their algorithm can only separate that component fingerprint which dominates the overlapped image. Overlapped fingerprint separation is analogous to the cocktail-party problem in automatic speech recognition [11]. Singh et al. [12] suggested the use of independent component analysis (ICA) to separate overlapped fingerprints, but they did not provide a separation algorithm. A common limitation of all these studies is that they were evaluated using a small number of example images and no objective evaluation was performed to determine whether the separation algorithms improved the matching accuracy, which is the ultimate goal.

In this paper, we present an algorithm to separate overlapped fingerprints and evaluate it using both real overlapped latent fingerprints and simulated overlapped fingerprints. The algorithm is based on the following two assumptions, which are both reasonable and practical:

1) The overlapped fingerprint image consists of at most two fingerprints. An overlapped fingerprint image with more than two component fingerprints is very difficult to separate even for fingerprint experts (see Fig. 2 for an example).

2) There are differences between the orientation fields of the two component fingerprints in the overlapped area. In other words, the components are identifiable.

The proposed algorithm consists of three steps:

1) An initial orientation field of the given overlapped image is estimated using local Fourier analysis [13]. The initial block-wise orientation field $O_{0}$ is an $m \times n \times 2(m \times n$ is the size of the block-wise image) matrix, recording at most two dominant orientations in each block.

2) A relaxation labeling method [14], [15] is employed to label the initial orientation field into two classes. Based on the labeling result, the initial orientation field is decomposed into two component orientation fields $O_{1}$ and $O_{2}$, each of size $m \times n$.

3) The two component fingerprints are separated by enhancing the overlapped fingerprint image using Gabor filters tuned to these two component orientation fields.

The flowchart of the proposed algorithm is shown in Fig. 3.

The rest of this paper is organized as follows. Section II estimates the initial orientation field of the given overlapped fingerprint image. In Section III, a relaxation labeling technique is proposed to decompose the initial orientation field. Section IV discusses the contextual enhancement scheme to separate the fingerprints based on the component orientation fields. Section V proposes an algorithm to utilize fingerprint

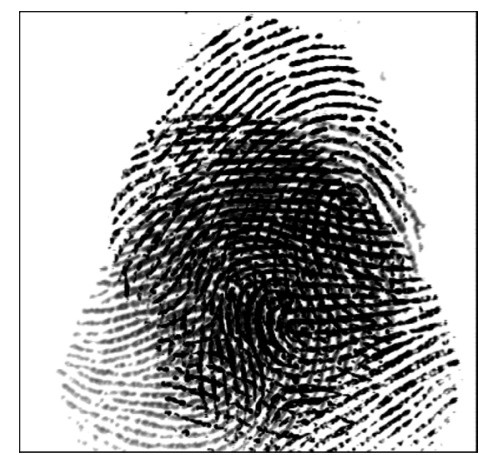

Fig. 2. Image with three overlapping fingerprints.

singularity information to improve the separation performance. Experimental results are presented in Section VI. We present conclusions and suggestions for future work in Section VII.

\section{Estimating Initial ORIENTATION FIELD}

A fingerprint orientation field is a matrix, whose value at $(x, y)$ denotes the dominant ridge orientation at point $(x, y)$ [2]. The orientation field of an overlapped fingerprint image is different from the orientation field of a single fingerprint image in that it contains one dominant orientation in the nonoverlapped regions and two dominant orientations in the overlapped region. In this study, we assume that the region masks of the component fingerprints in the given overlapped fingerprint image have been manually marked. ${ }^{1}$ The overlapped region can be easily obtained by finding the intersection of two fingerprint region masks. Therefore, the problem of initial orientation field estimation is to estimate one dominant ridge orientation in nonoverlapped fingerprint regions and two dominant ridge orientations in the overlapped region. The initial orientation field together with region masks provide all the information which is required by the subsequent orientation field separating algorithm.

Traditional orientation field estimation algorithms [2], [16], [17] consist of two steps: initial estimation (i.e., using a gradient-based method), followed by orientation field regularization. Regularization may be done by a simple averaging filter or complicated global model-based methods [16], [17]. But, for overlapped fingerprints which have ridges of two different orientations in the overlapped area, the initial orientation field obtained by gradient-based methods may be a random mix of the orientation fields of the two component fingerprints which cannot be resolved by existing regularization algorithms.

In order to extract the orientation fields of the two component fingerprints in the overlapped region, we use the local Fourier analysis method [13] to estimate the initial orientation field. An overlapped fingerprint image $I(x, y)$ is divided into nonoverlapping blocks of $16 \times 16$ pixels. Since the ridge structure in a block can be approximated by a 2-D sine wave, the task of estimating local ridge orientation is transformed to estimating the parameters of sine wave in each block. Centered at each block, the local image in the $64 \times 64$ window is multiplied

\footnotetext{
${ }^{1}$ Manually marking the region mask is a common practice in the latent fingerprint community, particularly for poor quality prints [4].
} 


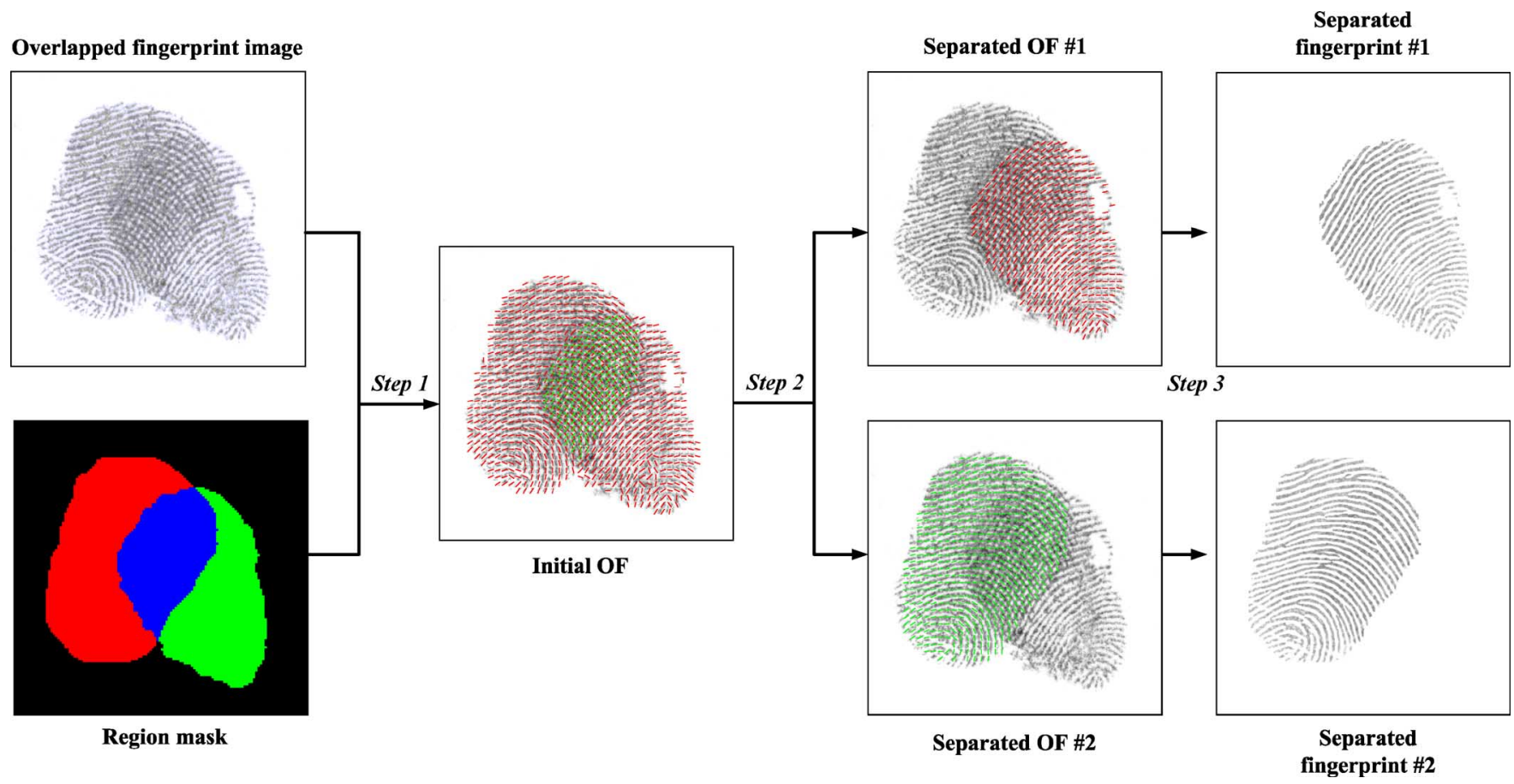

Fig. 3. Proposed algorithm for separating overlapped fingerprints: 1) estimating the initial orientation field (OF); 2) separating overlapped orientation fields into component orientation fields; and 3) separating overlapped fingerprints into component fingerprints.

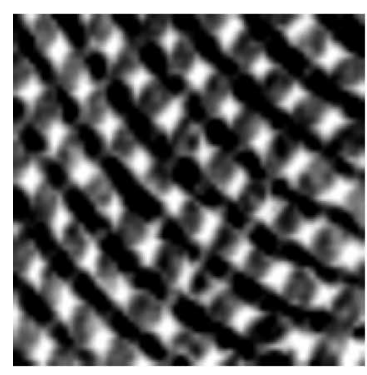

(a)

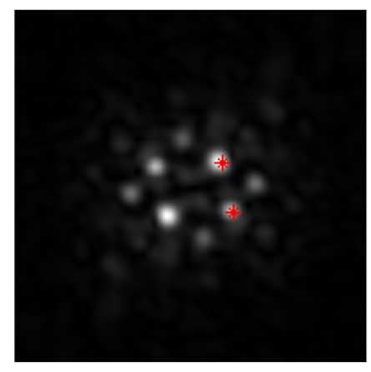

(b)

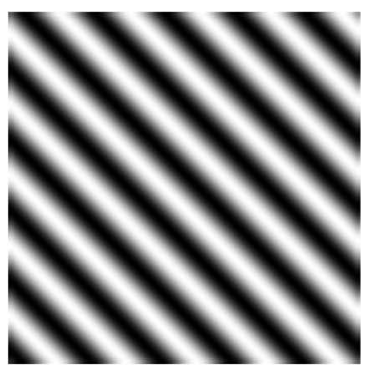

(c)

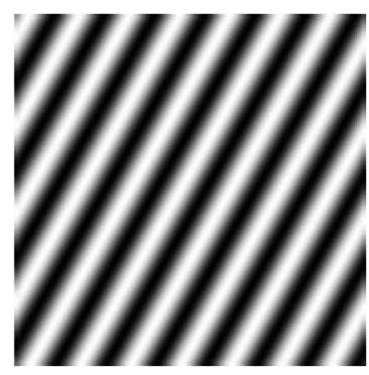

(d)

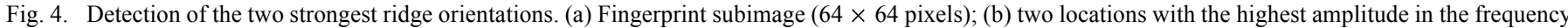

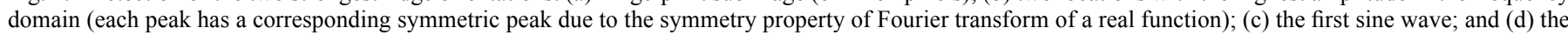
second sine wave.

by a bivariate isotropic Gaussian function $(\sigma=16)$. The discrete Fourier transform (DFT) $F(u, v)$ of the resulting image is computed and the amplitude of low-frequency components (points within three pixels from the center in the frequency domain) is set to 0 . In the frequency domain, one or two (one for the nonoverlapped region and two for the overlapped region) local maxima points with the largest amplitude are found. Each of these points corresponds to a 2-D sine wave $\omega(x, y)=$ $a \cdot \sin (2 \pi f(\sin (\theta) x+\cos (\theta) y)+\Phi)$, where $a, f, \theta$, and $\Phi$ represent the amplitude, frequency, orientation, and phase, respectively. These steps are illustrated in Fig. 4.

The initial orientation field consists of two orientations in each overlapped block and one orientation in each nonoverlapped block (as shown in Fig. 5). Fig. 6 shows that the amplitude of sine waves is not effective for separating the two orientation fields, since the ridge pattern of one fingerprint is not always stronger than the other in the overlapped region. To better separate overlapped orientation fields, it is necessary to utilize contextural information.

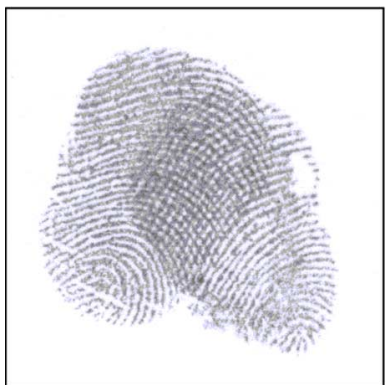

(a)

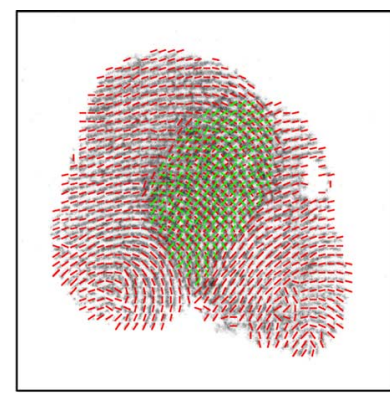

(b)
Fig. 5. Computing the initial orientation field. (a) Overlapped fingerprint image; (b) the initial orientation field of (a). Note that two different orientations are present in the overlapped region which are more visible in Fig. 6. In the overlapped region, orientations with the larger amplitude are marked in red while orientations with the smaller amplitude are marked in green. There is only one dominant orientation (marked in red) in the nonoverlapped regions.

\section{SePARATING OVERLAPPED ORIENTATION FIELD}

The initial orientation field $O_{0}$ contains two dominant orientations in each $16 \times 16$ overlapped block. We now propose a relax- 


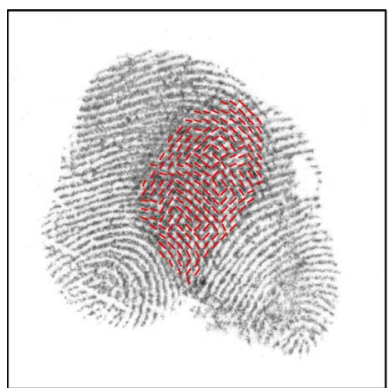

(a)

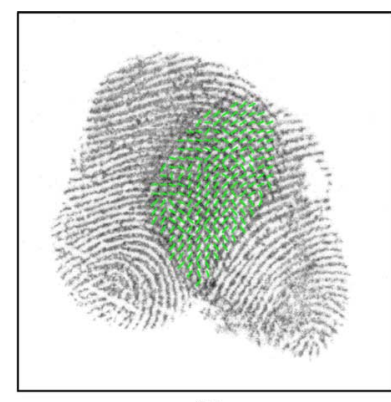

(b)
Fig. 6. Directly separating the orientation field of Fig. 5(b) using the amplitude of sine waves is not successful: (a) orientation with larger amplitude and (b) orientation with smaller amplitude.

ation labeling algorithm to separate it into two different orientation fields which correspond to the two component fingerprints.

\section{A. Relaxation Labeling}

Relaxation labeling is a generic name for a family of iterative algorithms which perform function optimization, based on local information [18]. It has been widely used in many different domains such as image processing, pattern recognition, and artificial intelligence [15]. See [19] and [20] for more details.

Relaxation labeling, as the name suggests, was proposed as a method for solving labeling problems in computer vision [21]. The relaxation labeling literature contains a variety of different algorithms (a survey and an extensive bibliography is given in [19]). In this paper, we use Rosenfeld et al.'s formulation [22].

Consider a labeling problem with $N$ objects $O=$ $\left\{o_{1}, o_{2}, \cdots, o_{N}\right\}$ and $M$ labels $\Lambda=\{1,2, \cdots, M\}$. A labeling is a function from the set of objects $O$ to the set of labels $\Lambda$. With each object $o_{i}$ by means of some local measurements we associate a probability vector $p_{i}=\left(p_{i 1}, p_{i 2}, \cdots, p_{i M}\right)^{T}$, where $0 \leq p_{i \lambda} \leq 1$, for $i=1,2, \cdots, N$, and $\lambda=1,2, \cdots, M$, $\sum_{\lambda} p_{i \lambda}=1$, for $i=1,2, \cdots, N$. Here, $p_{i \lambda}$ is the probability with which label $\lambda$ is associated with object $o_{i}$. Let $P=\left(p_{1}, p_{2}, \cdots, p_{N}\right)$ denote a label assignment of $N$ objects.

It is assumed that object labels do not occur independently of each other. The domain knowledge relevant to the problem is specified through a set of compatibility functions, $R_{i j}: \Lambda \times$ $\Lambda \rightarrow R, i=1,2, \cdots, N$, and $j=1,2, \cdots, N$. It is a $M \times M$ matrix defined as

$$
R_{i j}=\left[\begin{array}{cccc}
R_{i j}(1,1) & R_{i j}(1,2) & \cdots & R_{i j}(1, M) \\
R_{i j}(2,1) & R_{i j}(2,2) & \cdots & R_{i j}(2, M) \\
\vdots & \vdots & \vdots & \vdots \\
R_{i j}(M, 1) & R_{i j}(M, 2) & \cdots & R_{i j}(M, M)
\end{array}\right] .
$$

$R_{i j}\left(\lambda, \lambda^{\prime}\right)$ can be thought of as the degree of compatibility (specified locally) between object-label pairs $\left(o_{i}, \lambda\right)$ and $\left(o_{j}, \lambda^{\prime}\right)$ : large values indicate high compatibility and small values indicate incompatibility. The collection of $R_{i j}$, $i=1,2, \cdots, N$, and $j=1,2, \cdots, N$ constitutes the $N \times N$ compatibility block matrix denoted as

$$
R=\left[\begin{array}{cccc}
R_{11} & R_{12} & \cdots & R_{1 N} \\
R_{21} & R_{22} & \cdots & R_{2 N} \\
\vdots & \vdots & \vdots & \vdots \\
R_{N 1} & R_{N 2} & \cdots & R_{N N}
\end{array}\right]
$$

Relaxation labeling computes the label assignment $P$ iteratively until it is convergent [23]. At the $t$ th iteration, a label probability vector $p_{i}(t)$ is associated with each object $o_{i}, i=$ $1,2, \cdots, N$. The process starts with some initial set of probabilities $P(0)$, obtained through noisy measurements on the objects. The algorithm specifies how the label probabilities are updated at each instant as summarized in Algorithm 1.

Algorithm 1: Relaxation Labeling Algorithm

Initialization: set $t=0$, obtain initial label probabilities:

$$
P(0)=\left(p_{1}(0), p_{2}(0), \cdots, p_{N}(0)\right) .
$$

while true do

// Selection of labels:

for $i=1,2, \cdots, N$ do

choose a label at random based on the current label probabilities $p_{i}(t)$.

end

// Calculation of responses:

for $i=1,2, \cdots, N$ do

Let $q$ be the label selected for $o_{i}$ in step 1; compute the response $\beta_{i q}$ to $o_{i}$ as

$$
\beta_{i q}=(1 / N) \sum_{j} R_{i j}\left(q, s_{j}\right),
$$
end

where $s_{j}$ is the label selected for object $o_{j}$ in step 1 .

\section{// Updating of label probabilities:}

for $i=1,2, \cdots, N$ do

Let $q$ be the label selected for $o_{i}$ in step $1, p_{i}(t)$ is updated as

$$
\begin{aligned}
& p_{i q}(t+1)=p_{i q}(t)+\alpha \beta_{i q}\left(1-p_{i q}(t)\right), \\
& p_{i r}(t+1)=p_{i r}(t)-\alpha \beta_{i q} p_{i r}(t), \quad r \neq q .
\end{aligned}
$$

\section{end}

// Iteration:

if probability vectors have converged then

break.

end

else

$t=t+1$.

end

end

\section{B. Separating Algorithm}

1) Problem Modeling: The initial orientation field $O_{0}$ is an $m \times n \times 2$ matrix. We treat every element $O_{0}(i, j, k), 1 \leq i \leq m$, $1 \leq j \leq n$, and $1 \leq k \leq 2$, as an object $o_{i \cdot n \cdot 2+j \cdot 2+k}$. Thus the object set is $O=\left\{o_{1}, o_{2}, \cdots, o_{m \cdot n \cdot 2}\right\}$, and the label set is $\Lambda=\{1,2\}$. To separate the initial orientation field $O_{0}$, we need to label each object of $O$ with exactly one label of $\Lambda$.

2) Building Compatibility Coefficients: It is widely known that the performance of relaxation process is greatly affected by the choice of compatibility coefficients. Since 


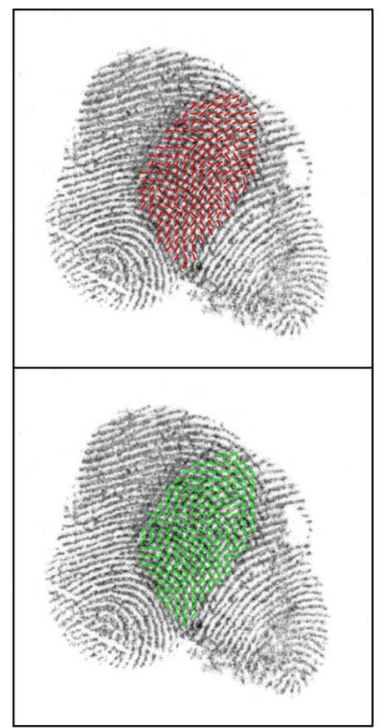

(a)

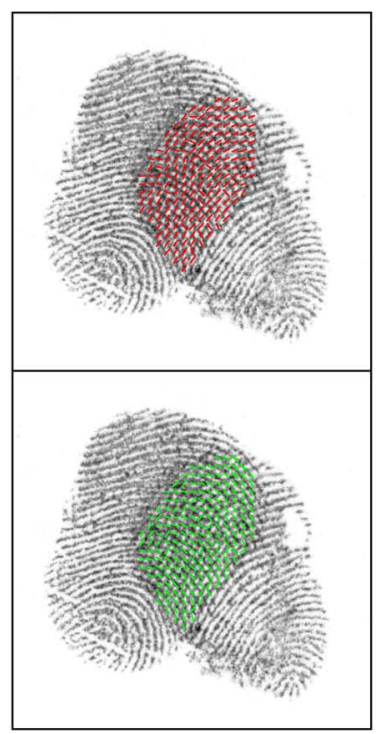

(b)

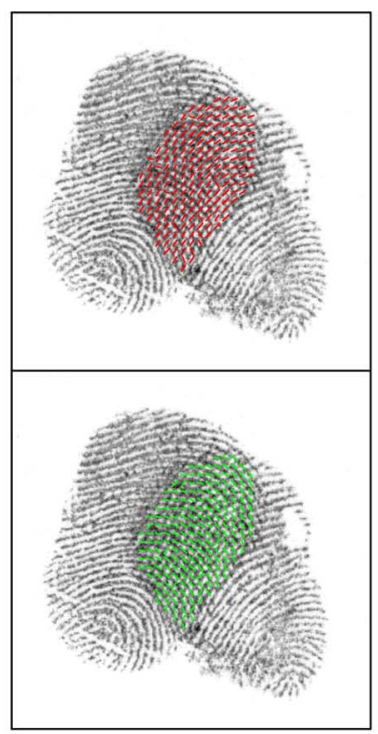

(c)

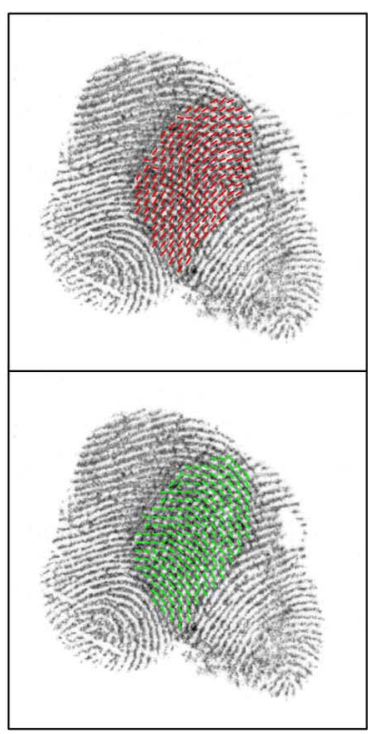

(d)

Fig. 7. Intermediate results of relaxation labeling for overlapping fingerprint image in Fig. 1(a). The two separated orientation fields are shown for different numbers of iterations (a) 60 , (b) 80 , (c) 100, and (d) 120 . The top row shows one of the separated orientation fields and the bottom row shows the other one.

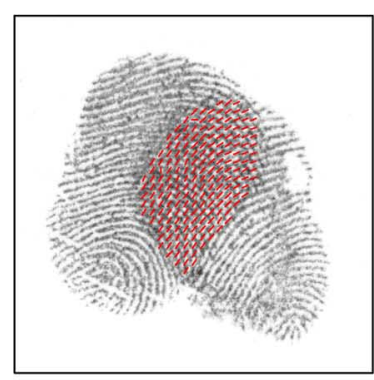

(a)

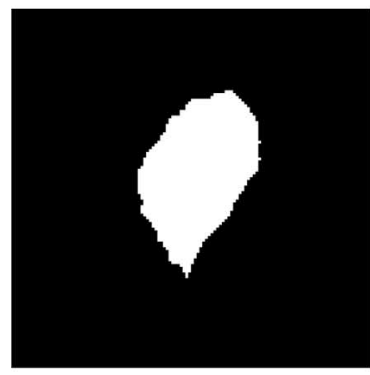

(e)

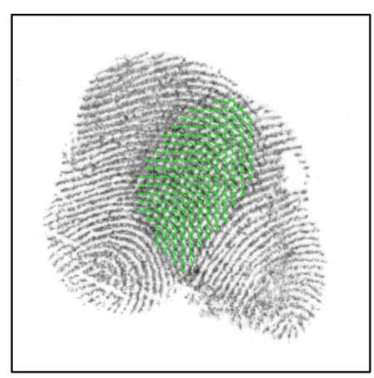

(b)

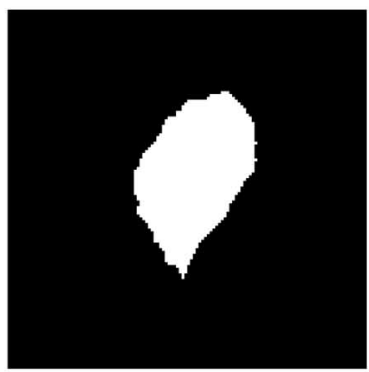

(f)

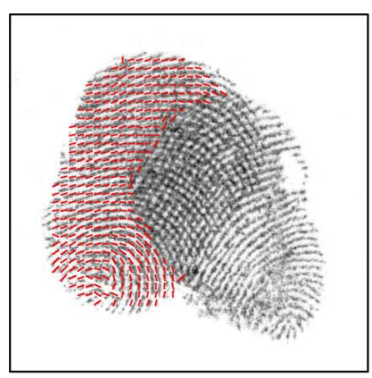

(c)

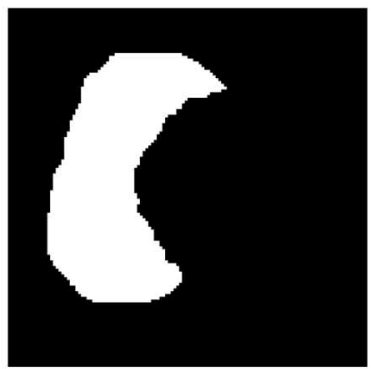

(g)

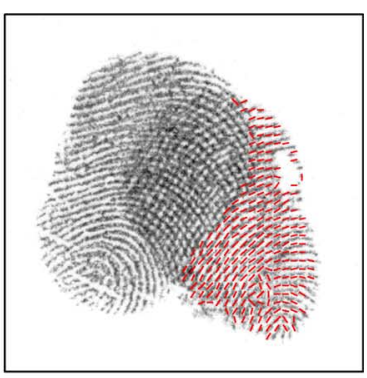

(d)

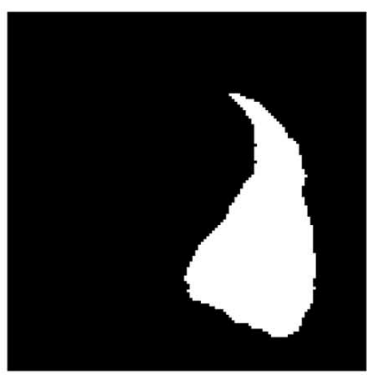

(h)

Fig. 8. Orientation fields in the overlapping and nonoverlapping regions. (a) The first separated orientation field in the overlapped region; (b) the second separated orientation field in the overlapped region; (c) one of the nonoverlapped orientation fields; (d) the other nonoverlapped orientation field. (e)-(h) are the corresponding binary region masks of (a)-(d), respectively.

relaxation labeling is based on local (contextual) information, an object $O(i, j, k)$ (namely $o_{i \cdot n \cdot 2+j \cdot 2+k}$; for clarity we use $O(i, j, k)$ instead of $\left.o_{i \cdot n \cdot 2+j \cdot 2+k}\right)$ is only supported by its neighborhood. The compatibility coefficient matrix $R_{I J}=R_{(i \cdot n \cdot 2+j \cdot 2+k)\left(i^{\prime} \cdot n \cdot 2+j^{\prime} \cdot 2+k^{\prime}\right)}$ between two objects $O(i, j, k)$ and $O\left(i^{\prime}, j^{\prime}, k^{\prime}\right)$ is defined as

$$
R_{I J}= \begin{cases}{\left[\begin{array}{ll}
0 & 0 \\
0 & 0
\end{array}\right],} & \text { if }\left|i-i^{\prime}\right|>D \text { or }\left|j-j^{\prime}\right|>D \\
{\left[\begin{array}{cc}
s & 1-s \\
1-s & s
\end{array}\right],} & \text { otherwise }\end{cases}
$$

where $s$ is the support when objects $O(i, j, k)$ and $O\left(i^{\prime}, j^{\prime}, k^{\prime}\right)$ have the same label, and $(1-s)$ is the support for different labels. In other words, $s$ is the support when orientations $O(i, j, k)$

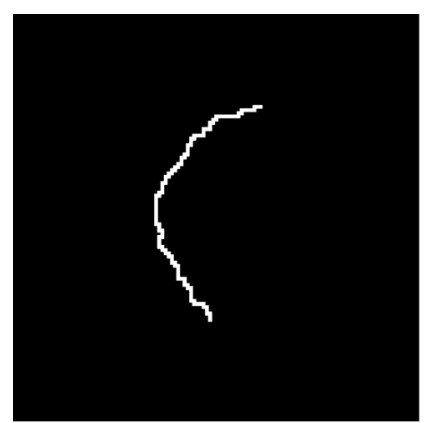

(a)

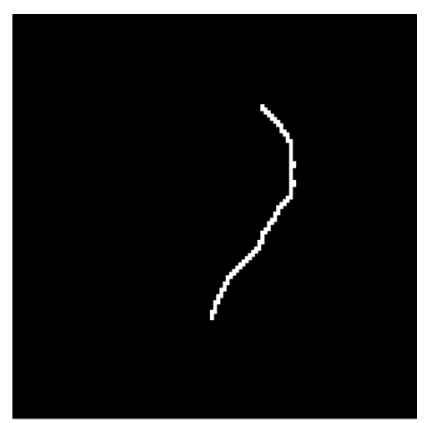

(b)
Fig. 9. (a) Boundary between the overlapped region and one of the two nonoverlapped regions of Fig. 1(a); (b) boundary between the overlapped region and the other nonoverlapped region. 


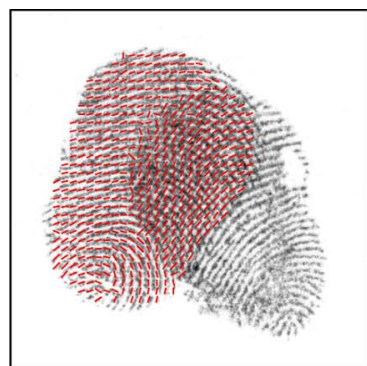

(a)

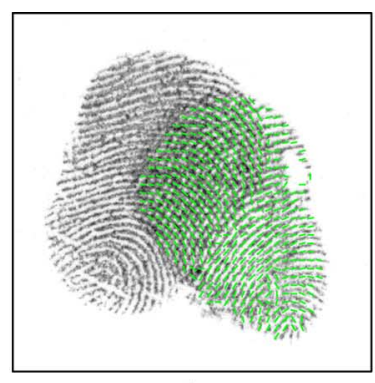

(b)

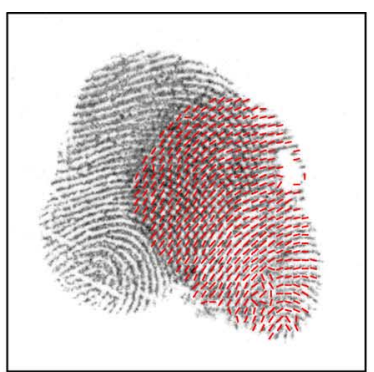

(c)

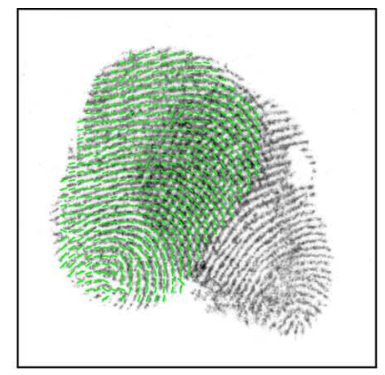

(d)

Fig. 10. Merging separated orientation fields in the overlapped region with the orientation fields in the nonoverlapped region. (a) and (b) correspond to one possible merger, while (c) and (d) correspond to another one.

and $O\left(i^{\prime}, j^{\prime}, k^{\prime}\right)$ come from the same fingerprint. Obviously, the smaller the difference between $O(i, j, k)$ and $O\left(i^{\prime}, j^{\prime}, k^{\prime}\right)$, the larger the $s$. Thus $s$ is computed as

$$
s=1-\frac{\delta\left(\left|O(i, j, k)-O\left(i^{\prime}, j^{\prime}, k^{\prime}\right)\right|\right)}{\pi / 2}
$$

where $O(i, j, k)$ and $O\left(i^{\prime}, j^{\prime}, k^{\prime}\right)$ are orientation values normalized to $(-\pi / 2, \pi / 2]$, and $\delta(\cdot)$ is defined as

$$
\delta(x)= \begin{cases}x, & \text { if } x \leqslant \frac{\pi}{2} \\ \pi-x, & \text { otherwise. }\end{cases}
$$

The purpose of the labeling is to obtain the two component orientation fields. Thus $O(i, j, 1)$ and $O(i, j, 2), 1 \leq i \leq m$, $1 \leq j \leq n$ should be set to different labels. Let $I=i \cdot n \cdot 2+$ $j \cdot 2+1$, and $J=i \cdot n \cdot 2+j \cdot 2+2$, then we have $R_{I J}=R_{J I}$ which are defined as

$$
R_{I J}=R_{J I}=\left[\begin{array}{ll}
0 & 1 \\
1 & 0
\end{array}\right]
$$

Equation (6) states that objects $O(i, j, 1)$ and $O(i, j, 2)$ have support 0 when they have the same label, and 1 for different labels.

Up to now, we have not discussed the compatibility coefficient matrix of an object $O(i, j, k)$ itself. Since an object itself has no information to support any label, $R_{I I}=R_{(i \cdot n \cdot 2+j \cdot 2+k)(i \cdot n \cdot 2+j \cdot 2+k)}$ is set as

$$
R_{I I}=\left[\begin{array}{ll}
0 & 0 \\
0 & 0
\end{array}\right] .
$$

To sum up, (3), (6), and (7) give the definition of compatibility coefficients.

It should be mentioned that the separation is only performed in the overlapped region due to the following two considerations. First, there is only one orientation in the nonoverlapped region. Second, processing only the overlapped region can save much computation time. Fig. 7 shows four intermediate separating results of Fig. 5(b) by relaxation labeling. Only the two orientation fields in the overlapped area are shown, with each column showing the intermediate steps. As can be observed, orientation field separation results improve gradually with the number of iterations. Using relaxation labeling, the initial orientation field in the overlapped area is correctly separated into two component orientation fields [Figs. 8(a) and (b)].

3) Merging Orientation Fields: The two separated orientation fields in the overlapped area should be merged with the

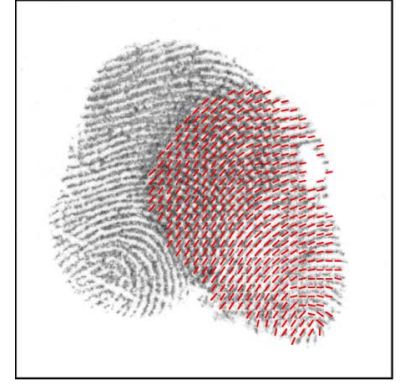

(a)

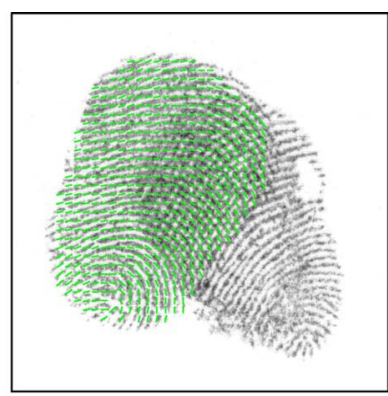

(b)
Fig. 11. Separated (component) orientation fields of Fig. 1(a).

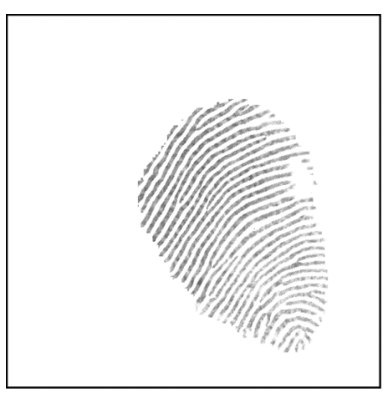

(a)

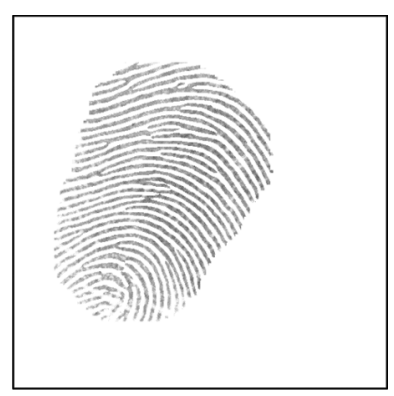

(b)
Fig. 12. Separating overlapped fingerprints of Fig. 1(a) by Gabor filtering: (a) the first enhanced fingerprints; (b) the second enhanced fingerprint.

two orientation fields in the nonoverlapped area to finalize the orientation field separation process. Fig. 8 shows the four orientation fields and the corresponding region masks. Denote the two overlapped orientation fields by $O_{o, 1}$ [Fig. 8(a)] and $O_{o, 2}$ [Fig. 8(b)], and the corresponding effective region by $M_{o}$ [Figs. 8(e) and (f)]. Here we use the suffix " $o$ " to indicate overlapped. Let $O_{n, 1}$ [Fig. 8(c)], and $O_{n, 2}$ [Fig. 8(d)] be the nonoverlapped orientation fields, and $M_{n, 1}$ [Fig. 8(g)], and $M_{n, 2}$ [Fig. 8(h)] be the corresponding region masks. Here we use the suffix " $n$ " to indicate nonoverlapped. By considering the continuity of orientation fields along the boundary of the overlapped region $M_{o}$, we merge the orientation fields which are more compatible with each other. The boundary $B_{j}$, $j=1,2$, between $M_{o}$ and $M_{n, j}$ is defined as

$$
B_{j}(x, y)= \begin{cases}1, & \text { if } M_{n, j}(x, y)=1 \text { and } \\ & \exists-D<\delta x<D,-D<\delta y<D \\ & M_{o}(x+\delta x, y+\delta y)=1 \\ 0, & \text { otherwise }\end{cases}
$$




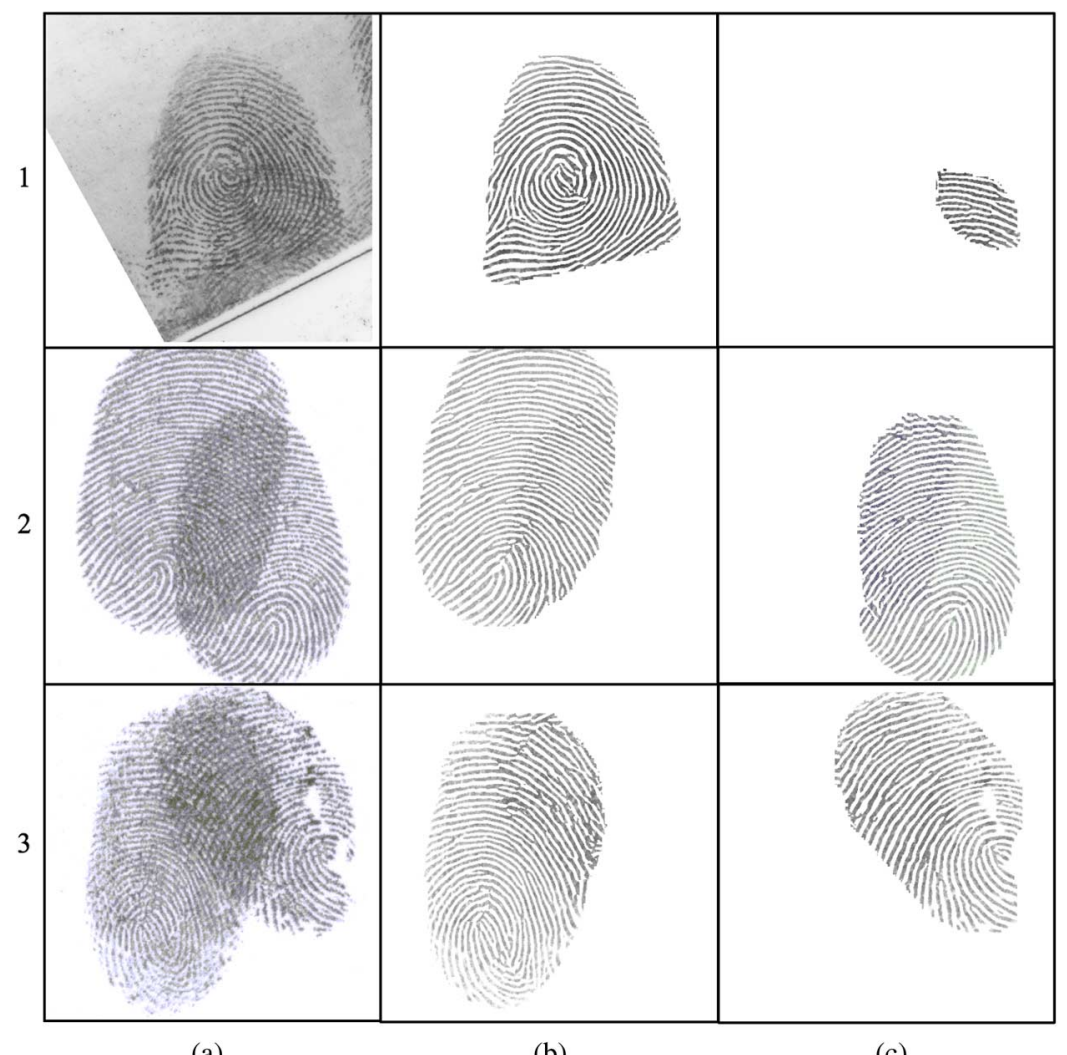

(a)

(b)

(c)

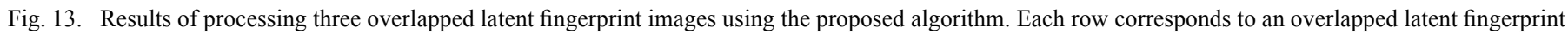
image. (a) Input overlapped latent fingerprint image; (b) first component fingerprint; (c) second component fingerprint.

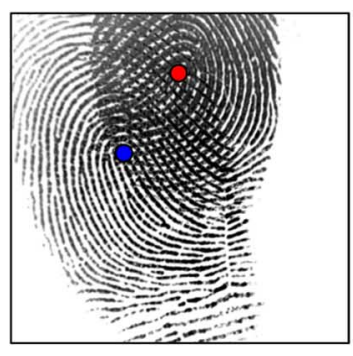

(a)

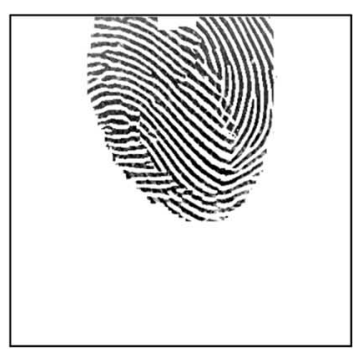

(b)

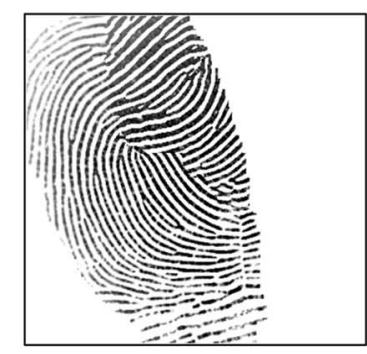

(c)

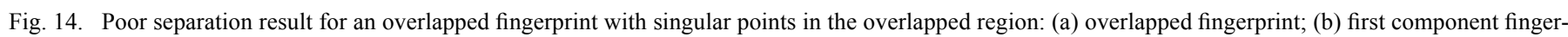
print; (c) second component fingerprint.

where $D$ is a parameter that controls the width of the boundary; in this study $D=6$ blocks. Fig. 9 shows the two boundaries of the overlapped fingerprint image in Fig. 1(a).

Now we try to find which one of the two possible combinations should be chosen to merge the orientation fields. The two possible combinations are: (i) $O_{o, 1}$ with $O_{n, 1}$ and $O_{o, 2}$ with $O_{n, 2}$, and (ii) $O_{o, 1}$ with $O_{n, 2}$ and $O_{o, 2}$ with $O_{n, 1}$, as shown in Fig. 10. For each combination, we compute the compatibility defined as

$$
\begin{aligned}
c_{1}=\frac{1}{2} & \frac{1}{N_{1}} \sum_{x} \sum_{y} \delta\left(\left|O_{o, 1}^{\prime}(x, y)-O_{n, 1}(x, y)\right|\right) \cdot B_{1}(x, y) \\
& \left.+\frac{1}{N_{2}} \sum_{x} \sum_{y} \delta\left(\left|O_{o, 2}^{\prime}(x, y)-O_{n, 2}(x, y)\right|\right) \cdot B_{2}(x, y)\right)
\end{aligned}
$$

and

$$
\begin{aligned}
c_{2}=\frac{1}{2}( & \frac{1}{N_{1}} \sum_{x} \sum_{y} \delta\left(\left|O_{o, 2}^{\prime}(x, y)-O_{n, 1}(x, y)\right|\right) \cdot B_{1}(x, y) \\
& \left.+\frac{1}{N_{2}} \sum_{x} \sum_{y} \delta\left(\left|O_{o, 1}^{\prime}(x, y)-O_{n, 2}(x, y)\right|\right) \cdot B_{2}(x, y)\right)
\end{aligned}
$$

where $O_{o, 1}^{\prime}$ and $O_{o, 2}^{\prime}$ are dilated [24] from $O_{o, 1}$ and $O_{o, 2}$, respectively, $\delta(\cdot)$ is defined in (5), and $N_{1}$ and $N_{2}$ are defined as

$$
N_{1}=\sum_{x} \sum_{y} B_{1}(x, y)
$$

and

$$
N_{2}=\sum_{x} \sum_{y} B_{2}(x, y) .
$$


If $c_{1}<c_{2}$, we choose the first combination; otherwise, choose the second combination. For this example, $c_{1}=0.58$, $c_{2}=0.19$, thus we choose the second combination as show in Figs. 10(c) and (d). After obtaining the two component orientation fields, an averaging filter is used to remove noise in each of the two orientation fields, and the resulting component orientation fields are shown in Fig. 11.

\section{SePARATING OverlapPed Fingerprints}

Given the component ridge orientation fields, we estimate the ridge frequency map of each component fingerprint using the method proposed in [3]. Given the ridge orientation field and ridge frequency map, Gabor filtering [25] can connect broken ridges and remove intervening ridges. Fig. 12 shows that this procedure successfully separates the two overlapping fingerprints.

Additional results on latent images containing overlapped fingerprints are shown in Fig. 13. Each row corresponds to an overlapped latent fingerprint image. The first column [Fig. 13(a)] is the overlapped latent fingerprint image. The second and third columns [Figs. 13(b) and (c)] show the two component fingerprints. All three examples of overlapped fingerprints shown in this figure have been successfully separated.

The above algorithm does not perform very well when the singularity region of component fingerprints is overlapped. An example is given in Fig. 14 to show this phenomenon. The underlying cause of this problem is that the relaxation labeling algorithm is solely based on local continuity of orientation field. As a result, it has a good labeling performance when the ridge orientation field of fingerprint is continuous. However, when the orientation field changes abruptly, for example near the singular points, it does not perform well. To make things more challenging, a part of the singularity region of one fingerprint may have an orientation field that is compatible with the other fingerprint.

We now explore how to utilize the fingerprint singularity information, if available, to assist in the orientation field separation.

\section{Utilizing Singular POINTS}

We assume that the singular points (core and delta) have been marked manually for each component fingerprint in the input overlapped area. Manual marking of singular points is a common practice in latent fingerprint community [4]. As proposed in [26], a fingerprint orientation field can be decomposed into singular orientation field and continuous orientation field

$$
O_{o}=O_{s}+O_{c} .
$$

The singular orientation field $O_{s}$ is defined by the Zero-Pole model proposed by Sherlock and Monro [27] as

$$
O_{s}=\frac{1}{2} \arg \left(\frac{\prod_{i}^{K}\left(z-z_{c_{i}}\right)}{\prod_{j}^{L}\left(z-z_{d_{j}}\right)}\right)
$$

where $z_{c_{1}}, \cdots, z_{c_{K}}, z_{d_{1}}, \cdots, z_{d_{L}}$ are the $K$ core and $L$ delta points in the fingerprint. The continuous orientation field is defined by

$$
O_{c}=O_{o}-O_{s}
$$

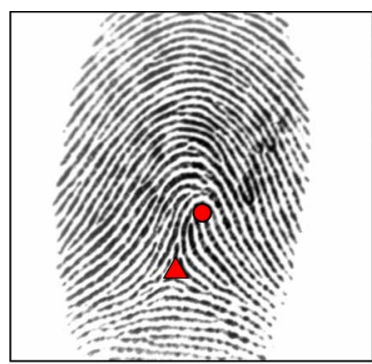

(a)

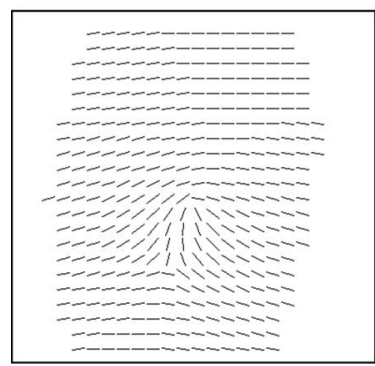

(c)

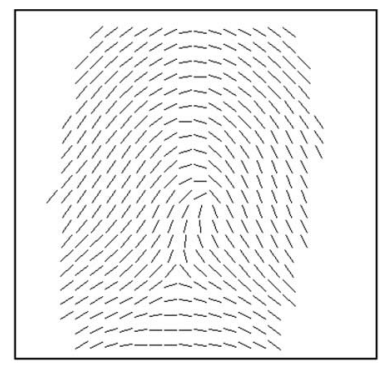

(b)

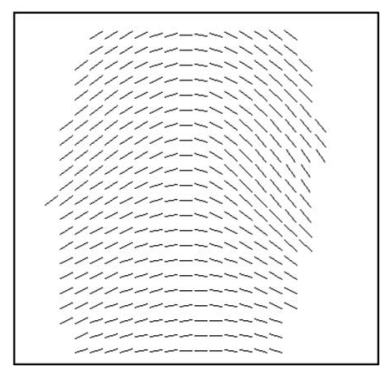

(d)
Fig. 15. Decomposition of orientation field: (a) fingerprint with marked singularities (circle indicates core and triangle indicates delta); (b) orientation field of (a); (c) singular, and (d) continuous orientation field components of (b).

Fig. 15 illustrates this decomposition of an orientation field. Note that the continuous orientation field is smooth everywhere. This suggests that we should use the continuous orientation field rather than the original orientation field for relaxation labeling. Singular points are incorporated into the relaxation labeling algorithm by modifying the compatibility coefficient matrix in (3). Suppose the singular orientation fields of two component fingerprints are denoted by $O_{s}(1)$ and $O_{s}(2)$. Then we have four possible continuous orientation fields $O_{c}\{k, l\}(k=1,2$, $l=1,2)$, as follows:

$$
O_{c}\{k, l\}=O_{0}(:,:, k)-O_{s}(l) .
$$

The compatibility coefficient matrix of (3) is changed to

$$
R_{I J}=\left\{\begin{array}{lc}
{\left[\begin{array}{ll}
0 & 0 \\
0 & 0
\end{array}\right],} & \text { if }\left|i-i^{\prime}\right|>D \text { or } \\
{\left[\begin{array}{ll}
s_{s}(1,1) & s_{s}(1,2) \\
s_{s}(2,1) & s_{s}(2,2)
\end{array}\right],} & \text { otherwise }
\end{array}\right.
$$

where $s_{s}\left(l, l^{\prime}\right)\left(l=1,2, l^{\prime}=1,2\right)$, is defined as

$$
s_{s}\left(l, l^{\prime}\right)=1-\frac{\delta\left(\left|O_{c}\{k, l\}(i, j)-O_{c}\left\{k^{\prime}, l^{\prime}\right\}\left(i^{\prime}, j^{\prime}\right)\right|\right)}{\pi / 2}
$$

where $\delta()$ is defined in (5). $O_{c}\{k, l\}(i, j)$ and $O_{c}\left\{k^{\prime}, l^{\prime}\right\}\left(i^{\prime}, j^{\prime}\right)$ are continuous orientation values normalized to $(-\pi / 2, \pi / 2]$.

The relaxation labeling algorithm now has better performance and provides better component fingerprints as shown in Fig. 16.

\section{EXPERIMENTAL RESULTS}

The ultimate goal of separating overlapped fingerprints is to successfully match the component fingerprint images to the 


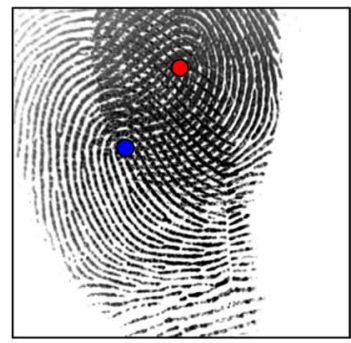

(a)

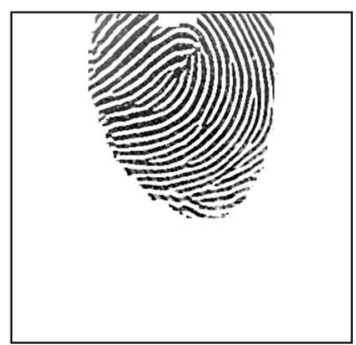

(b)

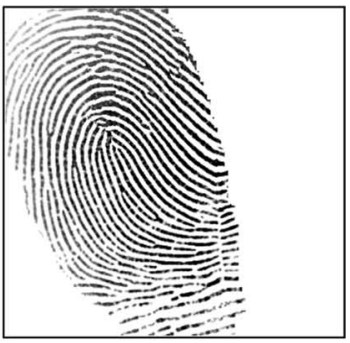

(c)

Fig. 16. Improved separation result by utilizing singular point information: (a) overlapped fingerprint; (b) first component fingerprint; (c) second component fingerprint.
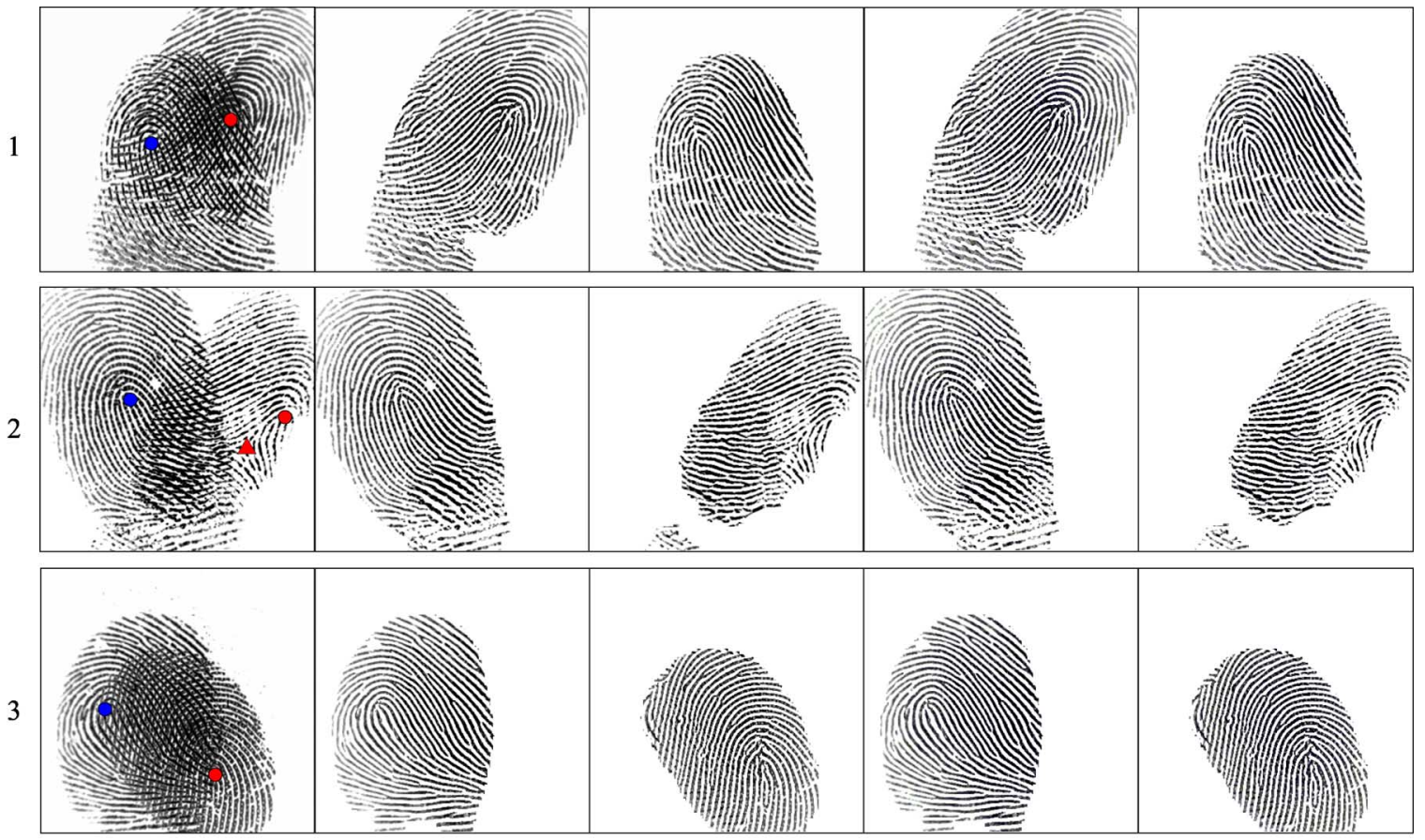

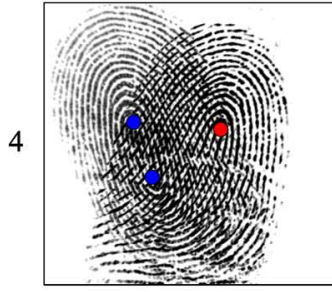

(a)

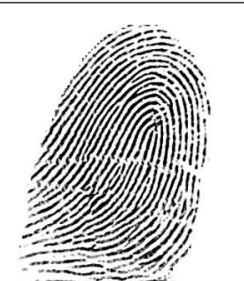

(b)

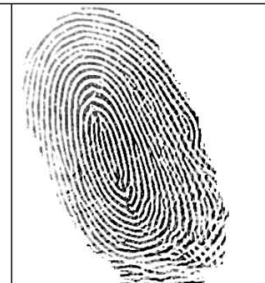

(c)

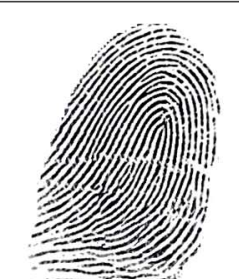

(d)

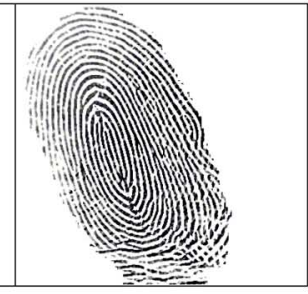

(e)

Fig. 17. Results of separating four simulated overlapped fingerprints with no significant improvement by using singular points. Each row corresponds to one example overlapped fingerprint. (a) Overlapped fingerprint image; (b) first component fingerprint image without using singular points; (c) second component fingerprint image without using singular points; (d) first component fingerprint image using singular points; (e) second component fingerprint image using singular points.

corresponding template fingerprints. While we have visually demonstrated the effectiveness of the proposed separating algorithm in preceding sections, we now conduct matching experiments to verify whether the component fingerprints obtained from the separating algorithm can indeed lead to higher matching accuracy than directly using the input overlapped fingerprint for matching.

We use two impressions (no. 3 and no. 4) of fingerprints in FVC2002 DB1 database to construct overlapped fingerprints and use impression no. 1 as the template fingerprint. A total of eight overlapped fingerprint images were simulated and classified into two groups. In the first group of four examples, the overlapped region does not contain any singularity or the singularities have little impact on the separating performance, while in the second group, singularities have a large impact on the separating performance. These two groups of examples and their separating results obtained by using the two separating algorithms (with/without singularity) are shown in Figs. 17 and 18. Each row corresponds to one example of overlapped fingerprint. The first column shows the simulated overlapped fingerprint images. The second and third columns contain the separated fingerprints without using the singularity information. The last two columns show the separated fingerprints when singularity information is utilized. 

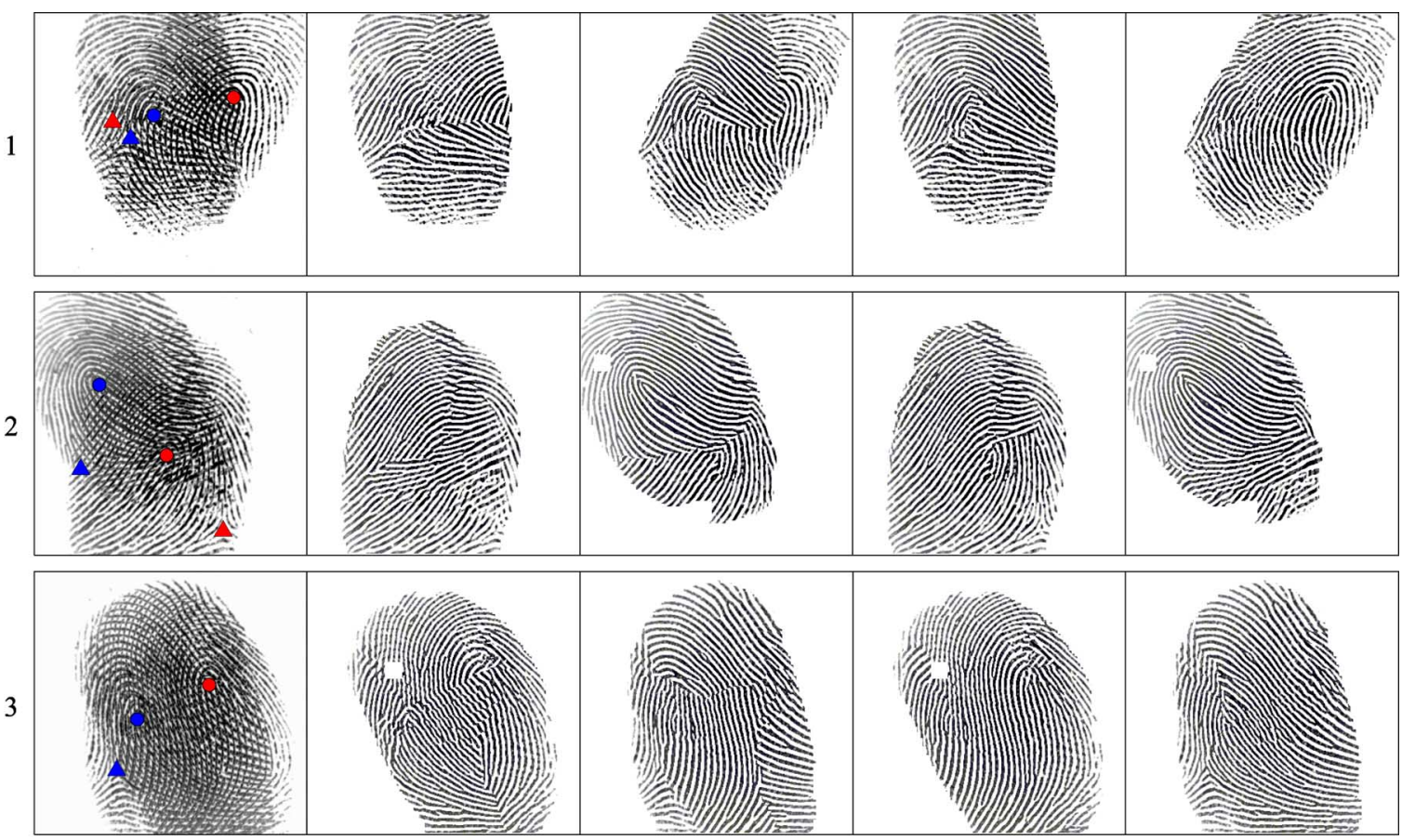

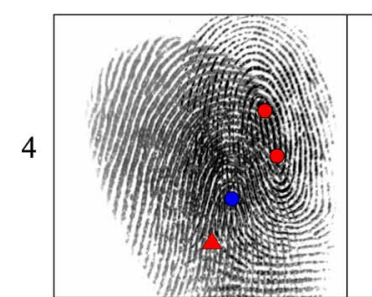

(a)

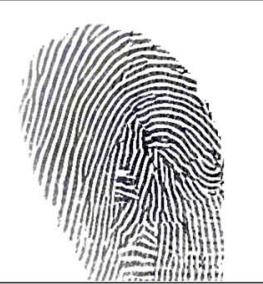

(b)

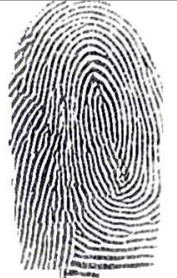

(c)

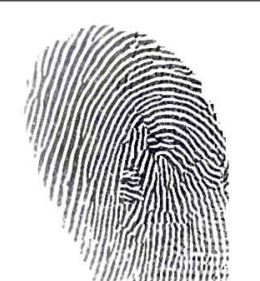

(d)

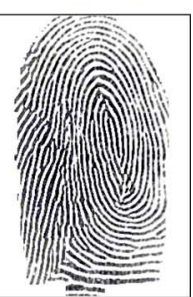

(e)

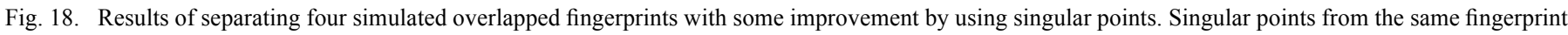

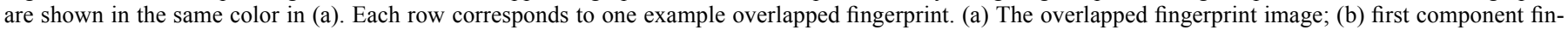

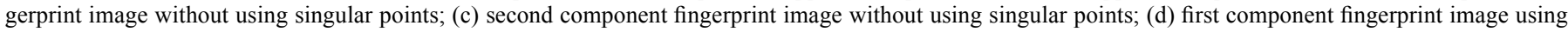
singular points; (e) second component fingerprint image using singular points.

Using the VeriFinger 6.2 SDK, two sets of matching experiments are performed for each overlapped image in the two groups. In the first set of experiments, the overlapped fingerprint, component fingerprint \#1 without using singularity, and component fingerprint \#1 using singularity are all matched to template fingerprint \#1. In the second set of experiments, the overlapped fingerprint, component fingerprint \#2 without using singularity, and component fingerprint $\# 2$ using singularity are all matched to template fingerprint $\# 2$. The eight matching scores (including the two matching scores between the original component fingerprint and the template fingerprint as a reference) for each of the four examples in the two groups are shown in Figs. 19 and 20.

The matching results for the first group in Fig. 19 show that the scores using separated fingerprints are generally higher than those obtained by directly using the overlapped fingerprint image except for one case (example \#2) where the separation algorithm using singularity leads to a slight drop in matching score. This illustrates the practical value of the proposed overlapped fingerprints separating algorithm. Since the singular points are at the edge of the overlapped area or outside the overlapped area, the impact of the singular points is small in this group of overlapped images. Thus the results of the two separating algorithms are quite similar, which can be seen in Fig. 19.

The second group of overlapped fingerprints is more challenging than the first group. The separation algorithm without singularity information leads to improvement in the matching scores of four component fingerprints, but reduces the scores of the other four component fingerprints. In contrast, the separation algorithm with singularity information improves the matching scores of seven of the eight component fingerprints. This demonstrates the effectiveness of the proposed separating algorithm using singularity information. The one case where both separation algorithms fail is case \#4 (the fourth row in Fig. 18). This is because in some regions of this image, the orientation fields of the two component fingerprints are not separable, which violates the assumption made in our separation algorithm.

We also applied our algorithm on four overlapped latent fingerprints (courtesy of Eric Widman, West Virginia University who collected these latents and their mated rolled fingerprints). These images are generally of poorer image quality than simulated ones. The separating results obtained by using the two algorithms (with/without singularity) are shown in Fig. 21. Each column corresponds to one overlapped latent image. 


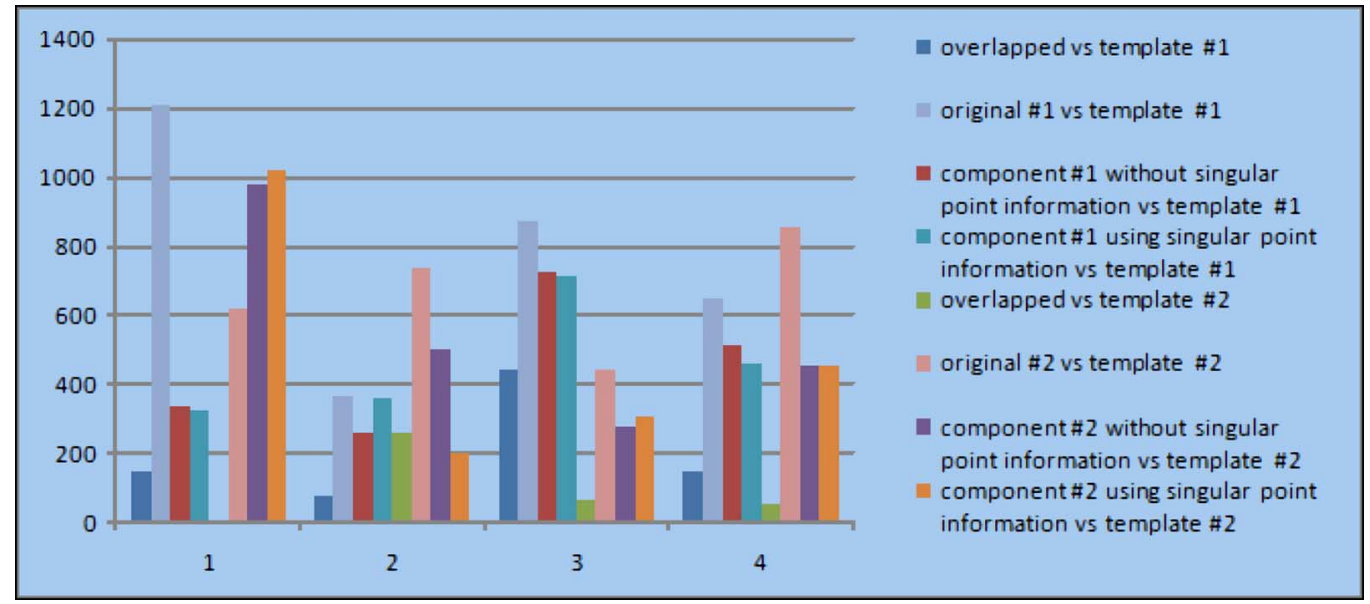

Fig. 19. Matching results (corresponding to Fig. 17) of the original fingerprint templates with the original component fingerprints, the overlapped fingerprint, and the two component fingerprints, respectively. Horizontal axis indicates the four overlapped examples in Fig. 17. Vertical axis shows the match score output by the VeriFinger matcher.

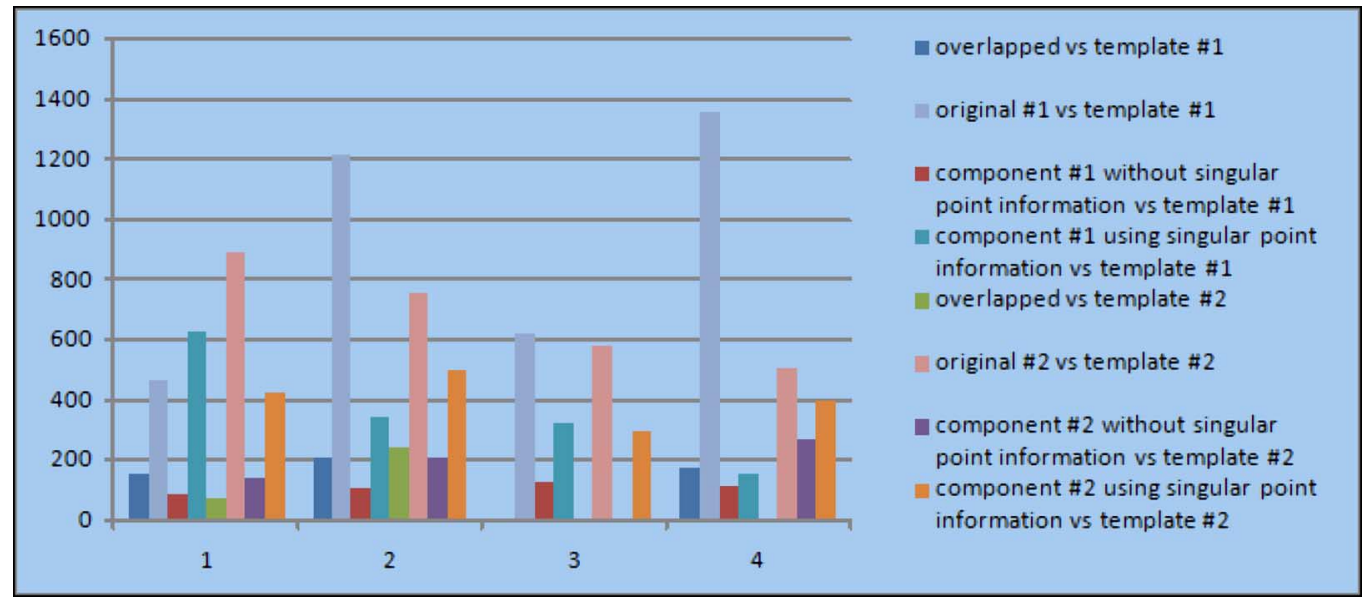

Fig. 20. Matching results (corresponding to Fig. 18) of the original fingerprint templates with the original component fingerprints, the overlapped fingerprint, and the extracted component fingerprints. Horizontal axis indicates the four examples in Fig. 18. Vertical axis shows the match score output by the VeriFinger matcher.

The first row shows the overlapped latent fingerprint images. The second and third rows contain the template fingerprints. The fourth and fifth rows contain the separated fingerprints without using the singularity information. The last two rows show the separated fingerprints when singularity information is utilized. The matching result in Fig. 22 shows that the proposed separating algorithm improves the matching scores of six component fingerprints. For the other two component fingerprints, there is no improvement in the matching scores. In the third example, since the two component orientation fields are almost the same (thus not separable) in the upper part of the fingers, only one component fingerprint is successfully separated.

The proposed separating algorithm was also tested on a larger dataset simulated using fingerprints in FVC2002 DB1_B. A total of 100 overlapped fingerprints were simulated by overlapping the impression no. 3 of each of the ten different fingers with the impression no. 4 of each finger. ${ }^{2}$ Before overlapping, the two images were rotated to meet the assumption that there are differences between the orientation fields of the two component

\footnotetext{
${ }^{2}$ These simulated overlapped fingerprint images are available at http://ivg.au. tsinghua.edu.cn/Datasets/Datasets.aspx.
}

fingerprints in the overlapped area. For each of the 100 overlapped fingerprints, VeriFinger matcher was used to match the overlapped image, and two separated fingerprints with/without singularity information to the other six impressions of the same finger. Note that no impostor matches have been conducted because the matching score of VeriFinger is linked to the false accept rate (FAR). Fig. 23 shows the receiver operating characteristic (ROC) curves of three types of matching scenarios. We can observe from the figure that the proposed separating algorithm significantly improves the matching accuracy of overlapped fingerprints. Further, the use of singularity information leads to better separating performance of several overlapped fingerprints.

\section{CONCLUSiOnS AND Future WORK}

\section{A. Conclusions}

Overlapped fingerprints are frequently encountered in latent fingerprint images collected at crime scenes. However, separating overlapped fingerprints is a very challenging problem for the state-of-the-art automatic fingerprint recognition systems as 
(a)

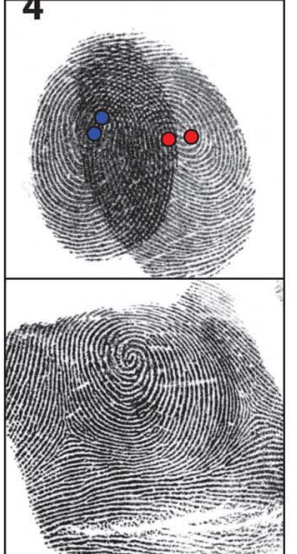

(c)

(d)

(e)

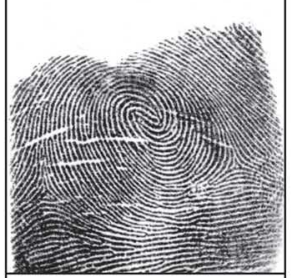

(f)
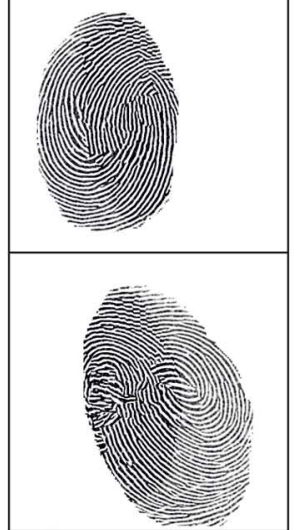

(g)

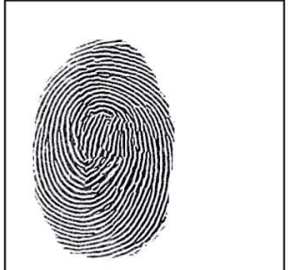

)

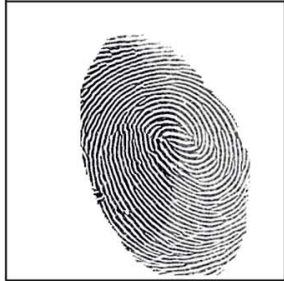

1

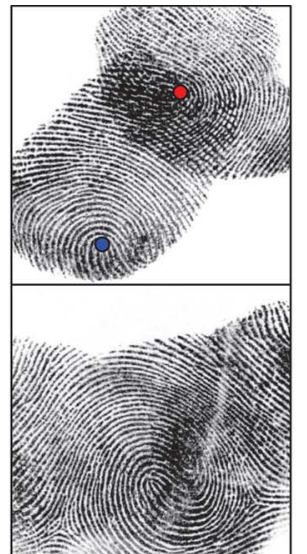

3
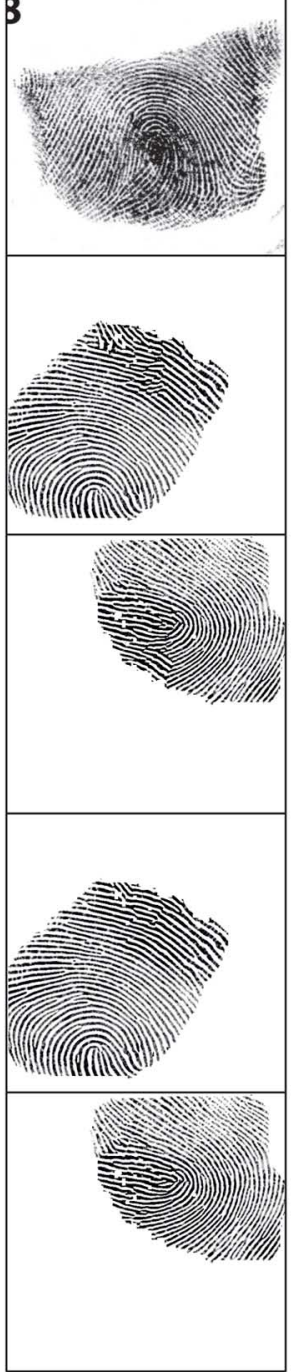

2

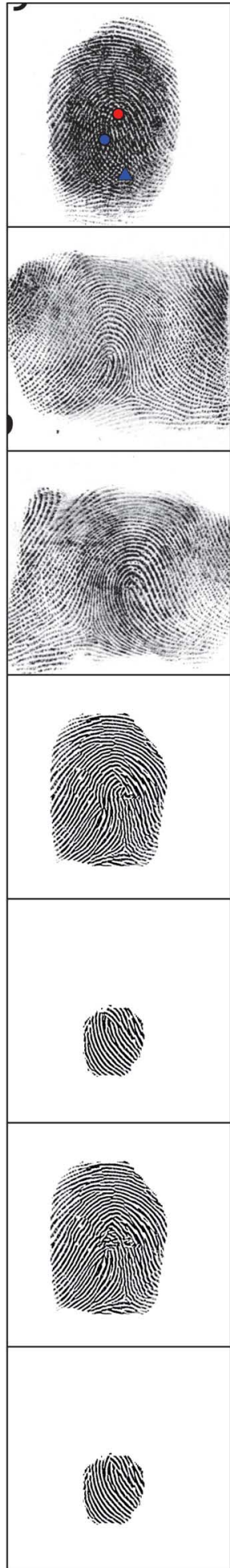

3

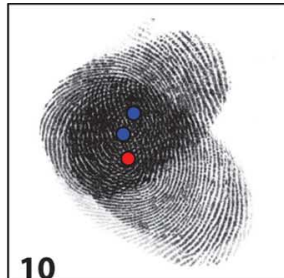

10

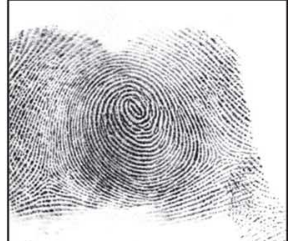

0

0
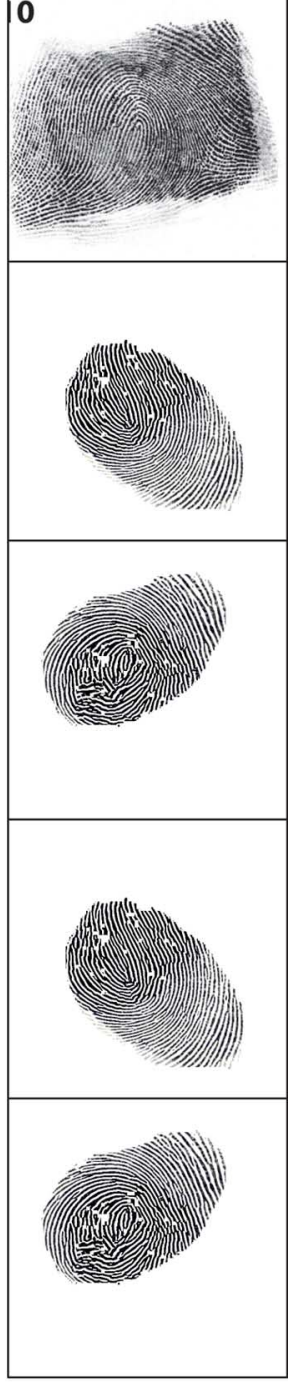

4

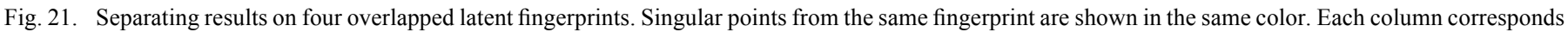

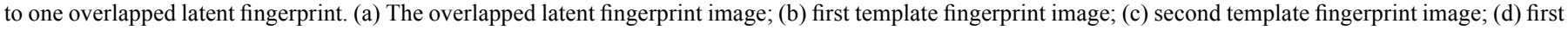

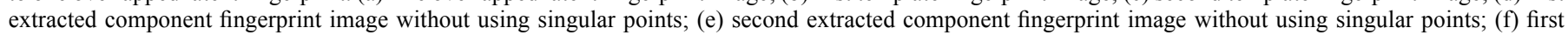
extracted component fingerprint image using singular points; $(\mathrm{g})$ second extracted component fingerprint image using singular points.

well as human fingerprint experts. We have proposed a novel algorithm for separating overlapped fingerprints. By applying a relaxation labeling method on the initial orientation field obtained by local Fourier analysis, we extract the two component orientation fields. The two component fingerprints are separated by filtering the overlapped fingerprint image using Gabor filters tuned to the component orientation fields. Satisfactory results were obtained on latent overlapped fingerprints. The proposed 


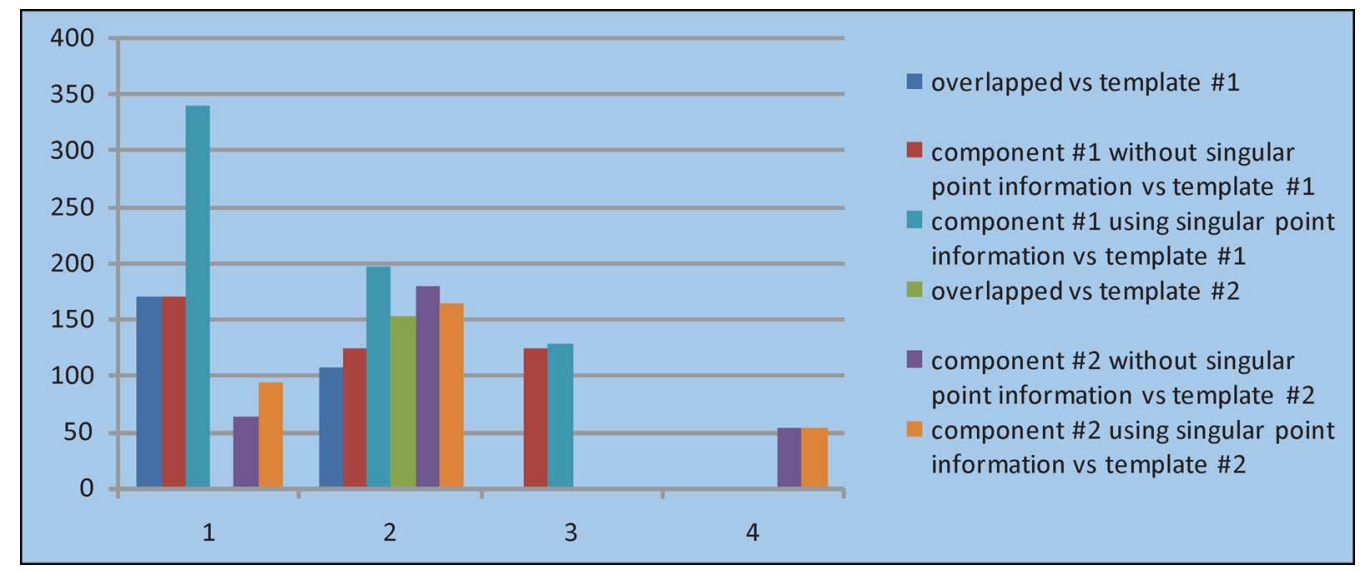

Fig. 22. Matching results (corresponding to Fig. 21) of the original fingerprint templates with the overlapped fingerprint, and the extracted component fingerprints. Horizontal axis indicates the four examples in Fig. 21. Vertical axis shows the match score output by the VeriFinger matcher.

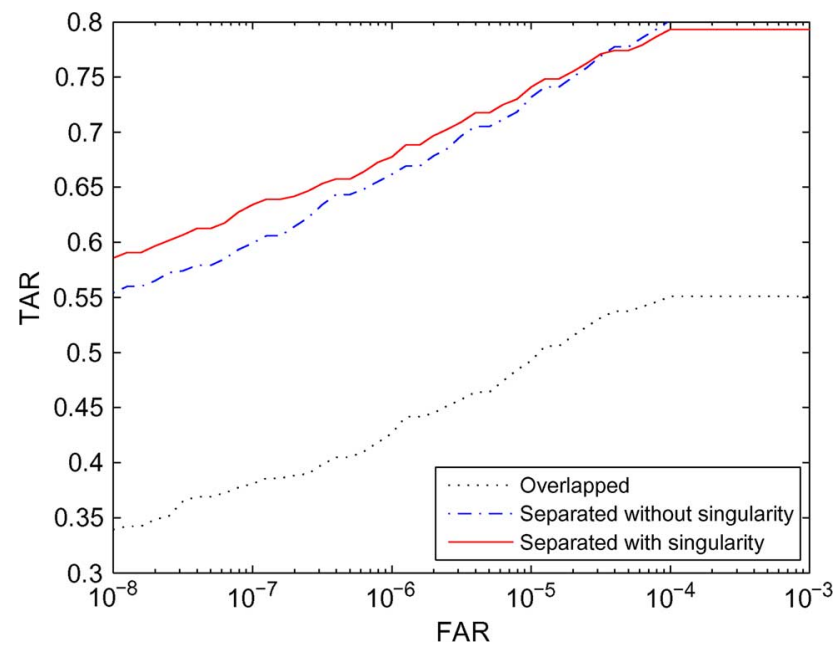

Fig. 23. ROC curves of using overlapped images and separated images with/ without singularity information.

algorithm will enable automatic fingerprint recognition systems to process and match overlapped fingerprints and will be a valuable tool for latent fingerprint examiners to mark features in latents.

\section{B. Future Work}

This study can be extended along the following directions: 1) The proposed algorithm assumes that the component orientation fields should be different or completely separable in the overlapped region. This may not always be the case. The algorithm needs to be improved to handle the more general case. 2) The current algorithm requires manually marked region masks (region of interest or ROI) and singular points as input. We plan to develop a fully automatic overlapped fingerprint separating algorithm. 3) Image quality of the overlapped fingerprints used in the current experiments is relatively good. We are in the process of collecting additional latent overlapped fingerprints of various quality that are lifted using different latent development methods.

\section{ACKNOWLEDGMENT}

The authors gratefully acknowledge the helpful comments of reviewers. They would also like to thank E. Widman at West Virginia University for providing some of the overlapped latent fingerprints.

\section{REFERENCES}

[1] F. Chen, J. Feng, and J. Zhou, "On separating overlapped fingerprints," in Proc. Fourth IEEE Int. Conf. Biometrics: Theory Applications and Systems (BTAS), 2010, pp. 1-6.

[2] D. Maltoni, D. Maio, A. K. Jain, and S. Prabhakar, Handbook of Fingerprint Recognition (Second Edition). New York: Springer, 2009.

[3] L. Hong, Y. Wan, and A. K. Jain, "Fingerprint image enhancement: Algorithm and performance evaluation," IEEE Trans. Pattern Anal. Mach. Intell., vol. 20, no. 8, pp. 777-789, Aug. 1998.

[4] A. K. Jain and J. Feng, "Latent fingerprint matching," IEEE Trans. Pattern Anal. Mach. Intell., vol. 33, no. 1, pp. 88-100, Jan. 2011.

[5] Neurotechnology ver. Verifinger 6.2, 2010, 2010 [Online]. Available: http://www.neurotechnology.com/

[6] S. Bramble and P. Fabrizi, "Observations on the effects of image processing functions on fingermark data in the Fourier domain," Proc. SPIE, vol. 2567, p. 138, 1995.

[7] W. J. Watling, "Using the FFT in forensic digital image enhancement," J. Forensic Identification, vol. 43, no. 6, pp. 573-584, 1993.

[8] H. Tang, W. Lu, C. Che, and K. Ng, "Gold nanoparticles and imaging mass spectrometry: Double imaging of latent fingerprints," Anal. Chem., vol. 82, no. 5, pp. 1589-1593, 2010.

[9] X. Fan, D. Liang, and L. Zhao, "A scheme for separating overlapped fingerprints based on partition mask," (in Chinese) Comput. Eng. Applicat., vol. 40, no. 2, pp. 80-81, 2004.

[10] R. Geng, Q. Lian, and M. Sun, "Fingerprint separation based on morphological component analysis," (in Chinese) Comput. Eng. Applicat., vol. 44, no. 16, pp. 188-190, 2008.

[11] A. Hyvärinen and E. Oja, "Independent component analysis: Algorithms and applications," Neural Netw., vol. 13, no. 4-5, pp. 411-430, 2000.

[12] M. Singh, D. Singh, and P. Kalra, "Fingeprint separation: An application of ICA," in Proc. SPIE, Mobile Multimedia/Image Processing, Security, and Applications, 2008, vol. 6982, pp. 69 820L-1-69 820L-11.

[13] A. K. Jain and J. Feng, "Latent palmprint matching," IEEE Trans. Pattern Anal. Mach. Intell., vol. 31, no. 6, pp. 1032-1047, Jun. 2009.

[14] M. Pelillo, F. Abbattista, and A. Maffione, "Evolutionary learning for relaxation labeling processes," in Proc. AI*IA, 1993, pp. 230-241.

[15] M. Pelillo and M. Refice, "Learning compatibility coefficients for relaxation labeling processes," IEEE Trans. Pattern Anal. Mach. Intell., vol. 16, no. 9, pp. 933-945, Sep. 1994.

[16] J. Zhou and J. Gu, "A model-based method for the computation of fingerprints' orientation field," IEEE Trans. Image Process., vol. 13, no. 6, pp. 821-835, Jun. 2004. 
[17] Y. Wang, J. Hu, and D. Phillips, "A fingerprint orientation model based on 2D Fourier expansion (FOMFE) and its application to singular-point detection and fingerprint indexing," IEEE Trans. Pattern Anal. Mach. Intell., vol. 29, no. 4, pp. 573-585, Apr. 2007.

[18] L. Padró, "Pos tagging using relaxation labelling," in Proc. Int. Conf. Computational Linguistics (COLING), 1996, pp. 877-882.

[19] J. Kittler and J. Illingworth, "Relaxation labelling algorithms-A review," Image Vision Comput., vol. 3, no. 4, pp. 206-216, 1985.

[20] A. J. Stoddart, M. Petrou, and J. Kittler, "On the foundations of probabilistic relaxation with product support," J. Math. Imag. Vision, vol. 9, no. 1 , pp. 29-48, 1998.

[21] A. Rangarajan, "Self-annealing and self-annihilation: Unifying deterministic annealing and relaxation labeling," Pattern Recognit., vol. 33, no. 4 , pp. $635-649,2000$

[22] A. Rosenfeld, R. A. Hummel, and S. W. Zucker, "Scene labeling by relaxation operations," IEEE Trans. Syst., Man, Cybern., vol. 6, no. 6, pp. 420-433, Jun. 1976.

[23] M. Thathachar and P. Sastry, "Relaxation labeling with learning automata," IEEE Trans. Pattern Anal. Mach. Intell., vol. 8, no. 2, pp. 256-268, Feb. 1986.

[24] R. M. Haralick and L. G. Shapiro, Computer and Robot Vision. Reading, MA: Addison-Wesley, 1992.

[25] J. Daugman, "Uncertainty relation for resolution in space, spatial frequency, and orientation optimized by two-dimensional visual cortical filters," J. Opt. Soc. Amer. A, vol. 2, no. 7, pp. 1160-1169, 1985.

[26] J. Feng, A. K. Jain, and A. Ross, "Detecting altered fingerprints," in Proc. 20th Int. Conf. Pattern Recognition (ICPR), 2010, pp. $1622-1625$

[27] B. G. Sherlock and D. M. Monro, "A model for interpreting fingerprint topology,” Pattern Recognit., vol. 26, no. 7, pp. 1047-1055, 1993.

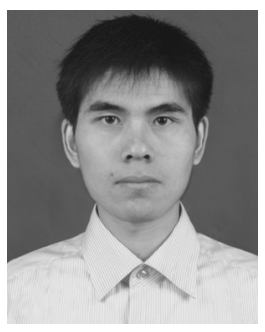

Fanglin Chen was born in 1983. He received the B.S. degree from the Department of Automation, Xi'an Jiaotong University, Xi'an, China, in 2006. Now he is working toward the Ph.D. degree in the Department of Automation, Tsinghua University, Beijing, China.

His research interests are in pattern recognition, image processing, and computer vision.

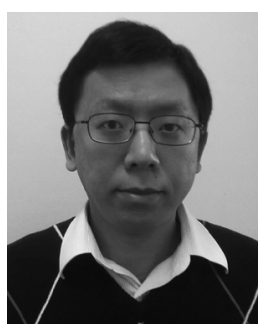

Jianjiang Feng (M'10) received the B.S. and Ph.D. degrees from the School of Telecommunication Engineering, Beijing University of Posts and Telecommunications, China, in 2000 and 2007, respectively.

$\mathrm{He}$ is an assistant professor in the Department of Automation at Tsinghua University, Beijing. From 2008 to 2009, he was a Postdoctoral researcher in the Pattern Recognition and Image Processing Laboratory at Michigan State University. His research interests include fingerprint recognition, palmprint recognition, and structural matching.

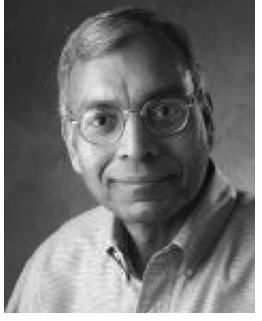

Anil K. Jain (S'70-M'72-SM'86-F'91) is a university distinguished professor in the Department of Computer Science and Engineering at Michigan State University, East Lansing. His research interests include pattern recognition and biometric authentication. He served as the Editor-in-Chief of the IEEE TRANSACTIONS ON PATTERN ANALYSIS AND MACHINE INTELLIGENCE (1991-1994). The holder of six patents in the area of fingerprints, he is the author of a number of books, including Handbook of Fingerprint Recognition (2009), Handbook of Biometrics (2007), Handbook of Multibiometrics (2006), Handbook of Face Recognition (2005), BIOMETRICS: Personal Identification in Networked Society (1999), and Algorithms for Clustering Data (1988). He served as a member of the Defense Science Board and The National Academies committees on Whither Biometrics and Improvised Explosive Devices.

Dr. Jain received the 1996 IEEE TRANSACTIONS ON NEURAL NETworks Outstanding Paper Award and the Pattern Recognition Society best paper awards in 1987, 1991, and 2005. He is a fellow of the AAAS, ACM, IAPR, and SPIE. He has received Fulbright, Guggenheim, Alexander von Humboldt, IEEE Computer Society Technical Achievement, IEEE Wallace McDowell, ICDM Research Contributions, and IAPR King-Sun Fu awards. ISI has designated him a highly cited researcher. According to Citeseer, his book Algorithms for Clustering Data (Englewood Cliffs, NJ: Prentice-Hall, 1988) is ranked \#93 in most cited articles in computer science.

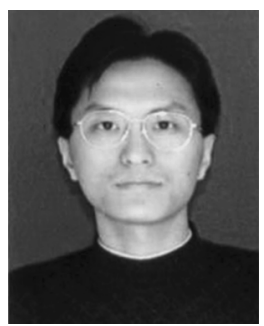

Jie Zhou (M'01-SM'04) was born in 1968. He received the B.S. and M.S. degrees from the Department of Mathematics, Nankai University, Tianjin, China, in 1990 and 1992, respectively, and the Ph.D. degree from the Institute of Pattern Recognition and Artificial Intelligence, Huazhong University of Science and Technology (HUST), Wuhan, China, in 1995.

Currently, he is a Full Professor with the Department of Automation, Tsinghua University. His research area includes pattern recognition, computer vision, and data mining. In recent years, he has authored more than 100 papers in peer-reviewed international journals and conferences.

Dr. Zhou received the Best Doctoral Thesis Award from HUST in 1995, First Class Science and Technology Progress Award from the Ministry of Education (MOE) in 1998, Excellent Teaching Award from Tsinghua University in 2003, and Best Advisor Awards from Tsinghua University in 2004 and 2005, respectively. He was selected as one of outstanding scholars of MOE in 2005. He is an Associate Editor for the International Journal of Robotics and Automation and Acta Automatica Sinica.

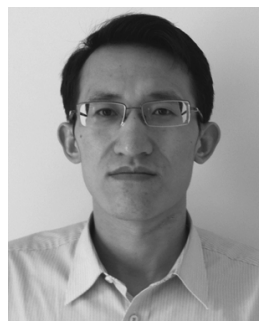

Jin Zhang was born in 1980 . He received the B.S. degree from the Department of Information Engineering, Nanjing University of Posts and Telecommunications, Nanjing, China, in 2002. Now he is working toward the Ph.D. degree in the Department of Automation, Tsinghua University, Beijing, China.

His research interests include pattern recognition, image processing, and computer vision. 